\title{
Diallel Crossing in Pinus cembra: V. Age Trends in Genetic Parameters and Genetic Gain for Height
}

\author{
By I. BladA ${ }^{1), 2)}$ and F. Popescu ${ }^{1)}$
}

(Received $11^{\text {th }}$ July 2011)

\begin{abstract}
A full diallel mating design (10 parents) was carried out in a Swiss stone pine panmictic population from the Carpathian Mountains. At age six, after nursery testing, the progenies were field planted to one site, using a completely randomized block design with 100 families, four replicates and a 15 tree row-plot per replication, spaced $2.5 \times 2.5 \mathrm{~m}$. Total and annual height of all individuals of this test was assessed at successive ages, between ages eight and 16. In addition, weight of 100 seeds and cotyledon number were considered in correlation analyses. Significant differences occurred in total height for general and specific combining ability effects. Variance components, heritabilities, genetic correlations and genetic gains on growth traits and survival at age 16 were estimated. Across the field testing period, $\mathrm{GCA}^{3)}$ variance increased with time, accounting for $65 \%$ of plot mean phenotypic variance by age 16 . In contrast, SCA variance declined as the progeny test aged accounting for only $9 \%$ by age 16 . The time trend of the four heritability estimates for total height increased with age across the testing period reaching their highest level between age 14 and 16. Genetic correlations over time for total height rose from 0.85 at age two to 0.94 at age six and then leveled off across the field test indicating that if the goal is to improve 16-year height, early selection can be considered at age six. By selecting the best $10 \%, 15 \%$ and $20 \%$ of individuals within the best fullsib families, a genetic gain in total height of $12.4 \%$, $11.0 \%$ and $9.9 \%$ respectively, could be achieved at age 16. A higher gain can be obtained if the best general combiner parents are selected and intermated.
\end{abstract}

Key words: Pinus cembra, diallel crossing, additive variance, dominance ratio, genetic effect, age-age genetic correlation, heritability, early selection, genetic gain.

\section{Introduction}

The Swiss stone pine (Pinus cembra L.) is a glacial relict species that occurs at high elevations in the Alps (RIKLI, 1909; CRITChFIELD and LITTLE, 1966) and the Carpathian Mountains (FEKETE and BlattNy, 1913; GEORGESCU and IONESCU-BARLAD, 1932) where it is found across the subalpine zone (HolzER, 1975). In the Alps, Swiss stone pine occurs between 1200 and 2500 m elevation, and is most frequent between 1500 and $2000 \mathrm{~m}$ (Contini and LAVARELlO, 1982). In the Romanian Carpathians the species ranges between $1350 \mathrm{~m}$ (BELDIE, 1940) and $2200 \mathrm{~m}$ (BLADA, 2007).

\footnotetext{
1) Forest geneticists, Forest Research and Management Institute Sos. Stefanesti, 128, Post Office 11, Bucharest, Romania.

2) Corresponding author: I. BLADA. E-mail: ioan_blada@icashd.ro.

${ }^{3}$ ) GCA and SCA refer to variances and g.c.a. and s.c.a. refer to effects (GRIFFING, 1956).
}

Swiss stone pine is important for several reasons (i) it has a major role in alpine forests, i.e. surviving in the harsh mountain climate and increasing the wind break and wind throw resistance of spruce in spruce-cembra mixed stands (BLADA, 1996), (ii) ecologically its crown density provides high capacity for retention of both fluid and solid precipitation (BLADA, 1996) providing stability in avalanche areas, and reducing flood effects, including soil erosion (HOLZER, 1969), (iii) its wood is useful for paneling and making traditional furniture, milk vessels and carvings (HOLTMEIER, 1994); sculpture for decorations of old churches (BRECHE and CHAVAUTIER, 1975) and for handicrafts manufacture (CONTINI and LAVARELLO, 1982), (iv) its high genetic resistance to blister-rust caused by Cronartium ribicola J.C. FISCH in RABENH. (BIngham, 1972; HoFF et al., 1980) (v) its ornamental use at low altitudes (HoLzER, 1969) and due to its variation in needle color, dense and conic-oval crown shape it is suitable in mountain landscaping, as well (BLADA, 1996).

Swiss stone pine is a slow growing species, but variation within this species was found (BLADA, 1999; 2003; 2008 ) even though variation was not observed in natural conditions. Owing to these encouraging results, land owners now want to plant deforested sloping mountainous lands and they are asking for improved material.

One of the aims of this breeding program is improving species growth rate and another is to test the selected material in harsh mountainous climatic conditions. In the frame of the previously mentioned program, a few results have been published on Swiss stone pine provenances (BLADA, 1997; 2007), half-sib families (BLADA, 1996; 2003) and full-sib families (BLADA, 1995; 1999; 2008). According to this authors' knowledge no other genetic improvement program with Swiss stone pine is still underway.

This paper provides new information about genetic and non-genetic parameters of growth and survival useful in breeding Swiss stone pine. In order to have a clear understanding of growth and its parameter estimates across the entire testing period of the trees, some nursery test data (BLADA, 1999) were considered in this analysis.

\section{Materials and Methods \\ Initial material, mating design and field design}

The 10 Swiss stone pine parent trees used in this study were selected as random samples from the Gemenele panmictic population located in the Retezat Massif of the Romanian southern Carpathians, at about $1800 \mathrm{~m}$ elevation. However, in order to have enough 
female strobili for pollination, reproductive fertility was taken into account in parent selection. To avoid relatedness, the distance between selected trees was at least $100 \mathrm{~m}$.

A $10 \times 10$ full-diallel mating design, according to GRIFFING (1956) Method 1, was carried out. Fresh pollen was used for all crossing. Controlled pollination took place by mid July, 1989, and seed was collected by the end of September, 1990. Pollen handling, bagging, pollination, and cone and seed handling were according to WRIGHT (1959). Nursery technique and testing procedures including the nursery results were presented by BLADA (1999).

Using the six year old progeny, a field trial was laid out in the Muncel zone of the Cibin Mountains, southern Carpathians, at about $1650 \mathrm{~m}$ elevation, $45^{\circ} 16^{\prime}$ north latitude and $24^{\circ} 31^{\prime}$ east longitudes. The 100 families (90 from out-crossing and 10 selfs) were planted out in a randomized complete block design with four replicates, with 15 trees row plots in each replicate, at $2.5 \times 2.5 \mathrm{~m}$ spacing.

\section{Measurements}

Total (H.8 to H.16) and annual height (h.9 to h.16) from ages eight to 16 , and survival at age 16 (SV.16) were recorded in the field progeny test. To avoid missing data because of missing trees in some families, only the first 10 living trees of the 15 trees planted in the row plots were measured.

\section{Linear model and statistical analysis}

Plot means of the 90 families (excluding the selfs) comprised the data set for statistical analysis according to GRIFFING (1956), Method 3, Model II where one set of $\mathrm{F}_{1}$ 's and reciprocals were included. However, the selfs were included in the progeny test in order to compare their performance with the outcrossed families (Figure 1).

The linear model used to estimate variance components for a modified full diallel laid out in a randomized complete block design in one location was a combination of GRIFFING (1956) and WILCOX (1976), such that:

$$
Y_{k i j}=u+b_{k}+g_{i}+g_{j}+s_{i j}+r_{j i}+m_{i}-m_{j}+n_{i j}+e_{k i j}
$$

where $Y_{k i j}=$ the mean performance in $k$-th block of the $i$-th female parent mated to the $j$-th male parent; $u=$ the population mean; $b_{k}=$ the effect of the $k$-th replication; $g_{i}$ and $g_{j}=$ the general combining ability effect for the $i$-th and $j$-th parents, respectively; $s_{i j}=$ the specific combining ability effect for the cross between the $i$-th and $j$-th parents such that $s_{i j}=s_{j i} ; r_{i j}=$ the reciprocal effect involving the reciprocal crosses between the $i$-th and $j$-th parents such that $r_{i j}=r_{j i} ; m_{i}$ and $m_{j}=$ the maternal effects of the $i$-th and $j$-th parents such that $m_{i}=m_{j}$; $n_{i j}=$ the interactions effect between $i^{\text {th }}$ and $j^{\text {th }}$ parents; $e_{k i j}=$ the random plot error variance associated with the kij-th individual observations. All effects except $u$ were assumed to be random variables and independent; also, the following restrictions are imposed on the combining ability effects: $\Sigma g_{i}=0$ and $\Sigma s_{i j}=0$ (for each j).

A general least squares analysis on plot mean basis was performed employing the computer program prepared by SCHAFFER and USANIS (1969), but the procedure was modified to permit separation of the maternal and maternal-interaction from the reciprocal variance. The analysis of the balanced modified full diallel was based upon the random model assuming that the parents were random samples from a random mating population. This assumption makes possible estimates of the genetic variance of the population. The model of analysis of variance, expected mean squares and formulas for estimating the variance components are listed in Table 1. The analysis of covariance was performed in an exactly analogous form, with the exception that mean cross products between ages were computed instead of mean squares for each age. All of the covariance components for each source of variance for each pair of ages were computed by the same least squares diallel computer program used to compute the variance components. Since data on individual trees were available, a separate analysis was performed in order to estimate the within plot variance $\left(\sigma_{\mathrm{w}}^{2}\right)(\operatorname{BECKER}, 1984)$. The $\sigma^{2}{ }_{\mathrm{w}}$ was estimated by pooling the among trees within plots variances.

Standard errors of variance components were computed by the ANDERSON and BANCROFT (1952) formula.

The component of variance $\sigma_{\text {GCA }}^{2}$ was used to estimate the variance in general combining ability among all of the parents in this experiment and is used as an estima-

Table 1. - Analysis of variance of modified full diallel, random effects model, in a randomized complete block layout (GRIFFING, 1956 combined with WILCOX, 1976).

\begin{tabular}{|c|c|c|c|}
\hline Source & Df & MS & $\mathrm{E} \cdot(\mathrm{VS})$ \\
\hline Rep. & $\mathrm{k}-1$ & $\mathrm{MS}_{\mathrm{Re}-\mathrm{i}}$ & $\sigma^{2} / n+\sigma_{p}^{2}+p(p-1) \sigma^{2}$ \\
\hline $\mathrm{GCA}$ & $\mathrm{p}-1$ & $\mathrm{MS}_{\mathrm{GCA}}$ & $\sigma^{2} \cdot \mathrm{nl} \sigma_{\mathrm{t}}^{2}+2 \mathrm{k} \sigma_{\mathrm{SCA}}^{2} \cdot 2 \mathrm{k}(\mathrm{p}-2) \sigma_{\mathrm{GCA}}^{2}$ \\
\hline$S C A$ & $p(p-3) / 2$ & $\mathrm{MS}_{\mathrm{scA}}$ & $\sigma^{2} \mathrm{n}+\sigma^{2}+2 \mathrm{k} \sigma^{2} \mathrm{son}$ \\
\hline Rec. & $p(p-1) / 2$ & $\mathrm{MS}_{\mathrm{Rec}}$ & $\sigma^{2} w / n+\sigma_{\Gamma:}^{2}+2 k \sigma_{k t: k}^{2}$ \\
\hline *Mat. & $\mathrm{p}-1$ & $\mathrm{MS}_{\text {Mat }}$ & 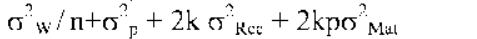 \\
\hline -Mat-Inleraction & $(p-1)(p-2) / 2$ & 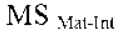 & 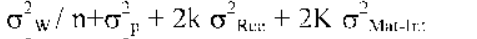 \\
\hline Error & $(k-1)\left(p^{2}-p-1\right)$ & $\mathrm{MS}_{\mathrm{E}:}$ & $\sigma^{2} w / n+\sigma_{F}^{2}$ \\
\hline Within plot & $k p(p-1)(n-1)$ & $\mathrm{MS}_{w}$ & $\sigma^{2}$ \\
\hline
\end{tabular}

$\sigma_{\mathrm{w}}^{2}=\mathrm{MS}_{\mathrm{w}} ; \sigma_{\mathrm{e}}^{2}=\mathrm{MS}_{\mathrm{E}} ; \sigma_{\mathrm{P}}^{2}=$ plot error $=\sigma_{\mathrm{e}}^{2}-\sigma_{\mathrm{w}}^{2} / \mathrm{n} ; \sigma_{\mathrm{Rec}}^{2}=\left(\mathrm{MS}_{\mathrm{Rec}}-\mathrm{MS}_{\mathrm{E}}\right) / 2 \mathrm{k}$;

$\sigma_{\text {Mat }}^{2}=\left(\mathrm{MS}_{\text {Mat }}-\mathrm{MS}_{\text {Mat-Int }}\right) / 2 \mathrm{kp} ; \sigma_{\text {Mat-Int }}^{2}=\left(\mathrm{MS}_{\text {Mat-Int }}-\mathrm{MS}_{\mathrm{E}}\right) / 2 \mathrm{k} ; \sigma_{\mathrm{SCA}}^{2}=\left(\mathrm{MS}_{\mathrm{SCA}^{-}} \mathrm{MS}_{\mathrm{E}}\right) / 2 \mathrm{k}$;

$\sigma_{\mathrm{GCA}}^{2}=\left(\mathrm{MS}_{\mathrm{GCA}}-\mathrm{MS}_{\mathrm{SCA}}\right) / 2 \mathrm{k}(\mathrm{p}-2) ; \mathrm{MS}_{\mathrm{Rep}}=\left(\mathrm{MS}_{\mathrm{Rep}}-\mathrm{MS}_{\mathrm{E}}\right) / 2 \mathrm{k}$;

$\mathrm{p}=$ progeny; $\mathrm{n}=$ seedlings per plot; $\mathrm{k}=$ replications. 
tor of $1 / 4 \sigma^{2}$. It was assumed that all epistatic components of genetic variance were insignificantly small and negligible. The component $\sigma_{\text {SCA }}^{2}$, or the estimated variance in specific combining ability, is an estimator of $1 / 4 \sigma_{D}^{2}$ assuming no inbreeding of the parents and no epistasis. Therefore, an estimate of the additive genetic variance was $4 \sigma_{\mathrm{GCA}}^{2}$ and an estimate of the dominance genetic variance was $4 \sigma_{\text {SCA }}^{2}$ (KRIEBEL et al., 1972; BECKER, 1984).

To estimate effectiveness of selection for traits analyzed, four heritabilities were calculated.

- The first heritability is the one commonly used for estimating the ratio of genetic (additive and non-additive) to total variance, which is appropriate for estimating gain from selection among families when they are vegetatively propagated. This broad-sense full-sib family heritability $\left(\mathrm{h}_{\mathrm{b}-\mathrm{sFS}}^{2}\right)$ was estimated according to the XIANG et al., 2003b) formula, adapted to this case, as:

$$
\begin{aligned}
\mathrm{h}_{\mathrm{b}-\mathrm{sFS}}^{2}= & \left(2 \sigma_{\mathrm{GCA}}^{2}+\sigma_{\mathrm{SCA}}^{2}\right) / \sigma_{\mathrm{Ph} 1}^{2}=\left(2 \sigma_{\mathrm{GCA}}^{2}+\sigma^{2}{ }_{\mathrm{SCA}}\right) / \\
& \left(2 \sigma_{\mathrm{GCA}}^{2}+\sigma_{\mathrm{SCA}}^{2}+\sigma_{\text {Mat }}^{2}+\sigma_{\text {Mat-Int }}^{2}+\sigma_{\mathrm{e}}^{2} / \mathrm{k}\right)
\end{aligned}
$$

where: $\sigma_{\text {GCA }}^{2}, \sigma_{\text {SCA }}^{2}, \sigma_{\text {Mat }}^{2}, \sigma^{2}{ }_{\text {Mat-Int }}$, are general, specific, maternal and maternal-interaction genetic variances, respectively; $\sigma_{\mathrm{e}}^{2}=$ environment variance; $\sigma_{\mathrm{Ph} 1}^{2}=$ plot mean phenotypic variance; $\mathrm{k}=$ number of blocks $=4$.

The mass selection genetic gain $\left(\Delta \mathrm{G}_{1}\right)$ according to FALCONER (1981) was estimated by:

$$
\Delta \mathrm{G}_{1}=\mathrm{i}_{1} \mathrm{~h}_{\mathrm{b} \text {-sFS }}^{2} \sigma_{\mathrm{Ph} 1}
$$

where: $i_{1}=$ the selection intensity taken from BECKER (1984); and $\sigma_{\mathrm{Ph} 1}=$ the phenotypic standard deviation of the selection unit. All genetic gains were estimated and expressed as percentage gain compared to the progeny test mean.

- The second heritability is that appropriate for estimating gain from selection among full-sib families when they are sexually propagated (individual selection). The narrow-sense full-sib family heritability $\left(\mathrm{h}^{2}{ }_{\mathrm{n} \text {-s FS }}\right)$ was estimated according to the (XIANG et al., 2003b) formula, adapted to this case, as:

$$
\begin{aligned}
\mathrm{h}^{2}{ }_{\mathrm{n}-\mathrm{sFS}}= & \left(2 \sigma_{\mathrm{GCA}}^{2}\right) / \sigma^{2}{ }_{\mathrm{Ph} 1}=\left(2 \sigma_{\mathrm{GCA}}^{2}\right) /\left(2 \sigma_{\mathrm{GCA}}^{2}+\right. \\
& \left.\sigma_{\text {SCA }}^{2}+\sigma_{\text {Mat }}^{2}+\sigma_{\text {Mat-Int }}^{2}+\sigma_{\mathrm{e}}^{2} / \mathrm{k}\right)
\end{aligned}
$$

and full-sib family selection genetic gain $\left(\Delta \mathrm{G}_{2}\right)$ was estimated by:

$$
\Delta \mathrm{G}_{2}=\mathrm{i}_{1} \mathrm{~h}_{\mathrm{n} \text {-sFS }}^{2} \sigma_{\mathrm{Ph} 1}
$$

- The third heritability is an individual tree narrowsense heritability ( $\mathrm{h}^{2}{ }_{\mathrm{n} \text {-s Ind }}$ ) (XIANG et al., 2003a) commonly used for estimating genetic gain from mass selection among randomly placed trees within the best families (full-sib in this case) of the progeny test, as:

$$
\begin{aligned}
\mathrm{h}^{2}{ }_{\mathrm{n} \text { - Ind }}= & \left(4 \sigma_{\mathrm{GCA}}^{2}\right) / \sigma_{\mathrm{Ph} .2}^{2}=\left(4 \sigma_{\mathrm{GCA}}^{2}\right) /\left(2 \sigma_{\mathrm{GCA}}^{2}+\right. \\
& \left.\sigma_{\mathrm{SCA}}^{2}+\sigma_{\text {Mat }}^{2}+\sigma_{\text {Mat-Int }}^{2}+\sigma_{\mathrm{p}}^{2}+\sigma_{\mathrm{w}}^{2}\right)
\end{aligned}
$$

where: $\sigma_{\mathrm{Ph} .2}^{2}=$ individual tree phenotypic variance; $\sigma^{2}=$ within plot variance; $\sigma^{2}=$ plot error variance $=\sigma^{2}{ }_{\mathrm{e}}-\sigma^{2}{ }_{\mathrm{w}} \mathrm{h}$; and $\mathrm{n}=$ seedlings per plot $=10$. by:

The mass selection genetic gain $\left(\Delta \mathrm{G}_{3}\right)$ was estimated

$$
\Delta \mathrm{G}_{3}=\mathrm{i}_{2} \mathrm{~h}_{\mathrm{n} \text {-s Ind }}^{2} \sigma_{\mathrm{Ph} .2}
$$

where: $i_{2}=$ the selection intensity for individual tree selection within full-sib families; $\sigma_{\mathrm{Ph} .2}=$ phenotypic standard deviation which refers to individual tree values.

- If using the previous heritability $\left(\mathrm{h}^{2}{ }_{\mathrm{n}-\mathrm{sFS}}\right)$ and selecting (through progeny testing) the best GCA parents from the base population and intermating, the selection intensity should be doubled (KRIEBEL et al., 1972; ZoBEL and TALBERT, 1984) to give the potential genetic gain $\left(\Delta \mathrm{G}_{4}\right)$ :

$$
\Delta \mathrm{G}_{4}=2 \mathrm{i}_{3} \mathrm{~h}_{\mathrm{n} \text {-sFS }}^{2} \sigma_{\mathrm{Ph} .1}
$$

The previously mentioned scenario was inserted because in the present progeny test, only two out of 10 randomly selected and tested parent trees proved to be good general combiners and they are not enough for further breeding work. Therefore, new candidates will be progeny tested in order to select GCA parents.

- The forth heritability is appropriate for estimating gain from selection among half-sib families when they are sexually propagated; this narrow-sense heritability $\left(\mathrm{h}^{2}{ }_{\mathrm{n}-\mathrm{sHS}}\right)$ is estimated (FALCONER, 1989) as:

$$
\begin{aligned}
\mathrm{h}^{2}{ }_{\mathrm{n}-\mathrm{sHS}}= & \left(\sigma^{2}{ }_{\mathrm{GCA}}\right) / \sigma^{2}{ }_{\mathrm{Ph} .3}=\left(\sigma_{\mathrm{GCA}}^{2}\right) /\left(\sigma_{\mathrm{GCA}}^{2}+\sigma^{2}{ }_{\mathrm{GCA}} /(\mathrm{p}-1)+\right. \\
& \left.\sigma_{\mathrm{SCA}}^{2} /(\mathrm{p}-1)+\sigma_{\text {Mat }}^{2}+\sigma_{\text {Mat-Int }}^{2}+\sigma^{2}{ }_{\mathrm{e}} / 2 \mathrm{k}\right)
\end{aligned}
$$

where: $p=$ the number of parent trees, i.e. 10 , and genetic gain $\left(\Delta \mathrm{G}_{5}\right)$ from half-sib family selection is estimated as:

$$
\Delta \mathrm{G}_{5}=\mathrm{i}_{4} \mathrm{~h}_{\mathrm{n} \text {-s HS }}^{2} \sigma_{\mathrm{Ph} .3}
$$

where: $i_{4}=$ selection intensity from 10 half-sib families; $\sigma_{\mathrm{Ph} .3}^{2}=$ plot mean phenotypic variance.

Standard errors (SE) for the heritability estimates were approximated by dividing the standard error of the $\sigma_{\text {GCA }}^{2}$ used as the numerator by the phenotypic variance of the selection unit used as the denominator (BECKER, 1984; HaLlauer and Miranda, 1981).

For genetic correlations $\left(r_{g}\right)$ estimates all of the covariance components for each source of variance for each pair of traits at different ages were computed by the same least squares diallel program used to compute the variance components (SCHAFFER and UsANIS, 1969; KRIEBEL et al., 1972), such that

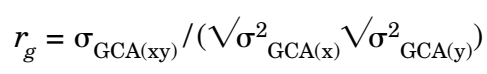

where: $\sigma_{\mathrm{GCA}(\mathrm{xy})}$ is the additive covariance component between traits $x$ and $y$ and $\sigma^{2}{ }_{\mathrm{GCA}(\mathrm{x})}$ and $\sigma_{\mathrm{GCA}(\mathrm{y})}^{2}$ are the variances due to GCA for traits $x$ and $y$, respectively.

General combining ability (g.c.a.) effects and their standard errors (S.E.) and the least significant differences $(L S D)$ were calculated according to GRIFFING (1956), Method 3, Model II.

In order to have a clear understanding of growth and its genetic and non genetic estimates across the whole living period of the trees, some nursery test data (BLADA, 1999) were added to the field data (see figures 1-, 2-, 3-, Table 6). As earlier in this chapter stated, the analysis of the balanced modified full diallel was performed employing the computer program prepared by SCHAFFER and USANIS (1969). 


\section{Results}

\section{Genetic variation}

The most important outcome of this experiment was that the analysis of plot means from the 90 outcrosses showed highly significant variation in general combining ability (g.c.a.) effects for both total and annual height (Table 2). Specific combining ability (s.c.a.) effects were significant or highly significant for all but two growth traits. With only one exception (h.10), significant and highly significant maternal effects occurred only after age 10. The maternal-interaction effects were significant or highly significant for total height, but insignificant for annual growth. Highly significant specific combining ability (s.c.a.) effects were found for survival at age 16, but no significant survival effects were found for g.c.a., maternal and maternal-interaction.
The above results suggested that though Swiss stone pine is an extremely slow growing species, it does possess high genetic variation in height growth, but not in survival. Growth traits are controlled by genes with both additive and dominance effects;

Comparisons of mean performance in total height between control-cross-pollinated (CP) and control-selfpollinated (SP) progeny are displayed in Figure 1. Throughout the evaluation of the experiment, mean height of outcrossed families exceeded the means of the selfed ones. The growth of both outcrossed and selfed families increased with age, but at different rates; the difference between them has reached $45.2 \%$ at age 16 . Because the selfed material tends towards inferiority with age, one may conclude that inbreeding depression is to be expected in this base population and it should be taken into account.

Table 2. - Analysis of variance of modified full diallel of the traits at different ages.

\begin{tabular}{|c|c|c|c|c|c|c|c|c|c|c|}
\hline \multirow[b]{2}{*}{ Source } & \multirow[b]{2}{*}{ D.E } & \multicolumn{9}{|c|}{ lraits : Mean squares } \\
\hline & & $\begin{array}{l}I .8 \\
(\mathrm{~cm})\end{array}$ & $\begin{array}{l}11.9 \\
\text { (cm) }\end{array}$ & $\begin{array}{l}\text { lI.10 } \\
(\mathrm{cm})\end{array}$ & $\begin{array}{l}1.11 \\
(\mathrm{~cm})\end{array}$ & $\begin{array}{l}{[1.12} \\
(\mathrm{cm})\end{array}$ & $\begin{array}{l}\mathrm{H} 1 . \mathrm{l} 3 \\
(\mathrm{~cm})\end{array}$ & $\begin{array}{l}1.14 \\
(\mathrm{~cm})\end{array}$ & $\begin{array}{l}I[.15 \\
(\mathrm{cm})\end{array}$ & $\begin{array}{l}11.16 \\
(\mathrm{~cm})\end{array}$ \\
\hline Rep & 3 & 73.35 & 93.38 & 64.87 & 289.35 & 564.91 & 283.18 & 124.19 & 341.06 & 38.86 \\
\hline GCA & 9 & $304.80 * * *$ & $486.37 * * *$ & $831.23 * * *$ & $992.86 * * *$ & $1907.56 * * *$ & $2835.22 * * *$ & $4045.67 * * *$ & $5328.56^{* * *}$ & $6511.96^{* * *}$ \\
\hline $\mathrm{SCA}$ & 35 & $40.68^{* * *}$ & $45.15^{* * *}$ & $82.19^{* * *}$ & $94.91 \div * *$ & $150.89^{* * * *}$ & $193.69 * * *$ & $253.86 * *$ & $394.71^{* * *}$ & $419.84^{* *}$ \\
\hline Rec. & 45 & $46.00^{* 2 * * 1}$ & $58.05^{\text {**** }}$ & $98.14^{* * *}$ & $95.27 * * *$ & $164.33^{* * *}$ & $228.54 * *$ & $296.12 * \cdots *$ & $336.86^{* * *}$ & $448.67 * * *$ \\
\hline - Mat & 9 & $85.25^{\circ}$ & 80.40 & 148.89 & $169.63^{2}$ & $351.78^{* *}$ & $514.58 * *$ & $683.34 * *$ & $654.89^{*}$ & $917.91 \%$ \\
\hline - Mat-Int & 36 & 36.18 **水 & $52.46^{* * * *}$ & $85.45 * * *$ & $76.68^{* * * *}$ & $117.46^{* *}$ & $157.04 * *$ & $199.32 \%$ & $257.36^{*}$ & $331.36^{*}$ \\
\hline T.rem & 26,7 & $9,(69)$ & 18.92 & 34.37 & 36,42 & 68,39 & 93,016 & $\mid 2 x, 07$ & 69.147 & 27,92 \\
\hline Mithin ylot & 3240 & 2668 & 48.81 & 84.34 & 117.50 & 185.98 & $340 . .5$ & 401.69 & $562.3:$ & 625,48 \\
\hline \multicolumn{11}{|c|}{ Table 2 continued } \\
\hline & & \multicolumn{9}{|c|}{ Traits Mcan squares } \\
\hline Source & D. $f^{\circ}$ & $\begin{array}{c}1.9 \\
(\mathrm{~cm})\end{array}$ & $\begin{array}{l}\text { h. } 10 \\
\text { (cm) }\end{array}$ & $\begin{array}{l}\mathrm{h} .11 \\
(\mathrm{~cm})\end{array}$ & $\begin{array}{l}\text { h. } 12 \\
\text { (cm) }\end{array}$ & $\begin{array}{l}\mathrm{h} .13 \\
(\mathrm{~cm})\end{array}$ & $\begin{array}{l}\text { h. } 14 \\
(\mathrm{~cm})\end{array}$ & $\begin{array}{l}\mathrm{h} .15 \\
(\mathrm{~cm})\end{array}$ & $\begin{array}{l}\text { h. } 16 \\
(\mathrm{~cm})\end{array}$ & $\begin{array}{c}\text { SV.16 } \\
(\operatorname{Arcsin} \%)\end{array}$ \\
\hline Rep & 3 & 7.08 & 5.93 & 49.46 & 25.52 & 37.05 & 22532 & 9.61 & 177.77 & 77.56 \\
\hline CiCA & 9 & $25.37 * * * *$ & $61.20 * * *$ & $41.62 * * *$ & $145.09 * * *$ & $186.87 * * *$ & $110.24 * * *$ & $149.72^{* * * *}$ & $164.12 * * *$ & 69.79 \\
\hline $\mathrm{SCA}$ & 35 & $5.70^{\text {* }}$ & $5.65^{*}$ & 4.14 & 7.46 & $14.81^{* 2 *}$ & $11.06 * *$ & $9.96^{* * h}$ & $10.85^{*}$ & $81.24 * *$ \\
\hline Ret. & 45 & 4.40 & 5.02 & 4.86 & 6.36 & $11.98 * *$ & $10.06^{* *}$ & $8.36 * *$ & $9.99 \%$ & 53.1 \\
\hline -Mat & 9 & 4.38 & 5.43 & $9.03 *$ & $12.73^{*}$ & $23.86^{*}$ & $20.23^{*}$ & $15.11 \%$ & $25.99^{*}$ & 57.33 \\
\hline - Vat-Int & 36 & 4.41 & 4.91 & 3.82 & 4.77 & 9.01 & 7.52 & 6.67 & 5.99 & 52.01 \\
\hline Error & 267 & 3.56 & 3.85 & 3.60 & 5.60 & 6.27 & 6.02 & 5.36 & 6.75 & 40.49 \\
\hline Within plot & 3240 & 10.11 & 11.34 & 13.70 & 18.54 & 23.08 & 15.13 & 14.03 & 15.20 & - \\
\hline
\end{tabular}

H. 8 to H.16 and h.9 to h.16 are the total and annual height between ages eight and 16, respectively; SV.16 is survival at age 16; ${ }^{*} \mathrm{p}<0.05 ;{ }^{* *} \mathrm{p}<0.01 ;{ }^{* * *} \mathrm{p}<0.001$. GCA, SCA, Rec, Mat and Mat-Int.= general, specific, reciprocal, maternal and maternalinteraction variances, respectively and all formulae for their calculations are given with Table 1.

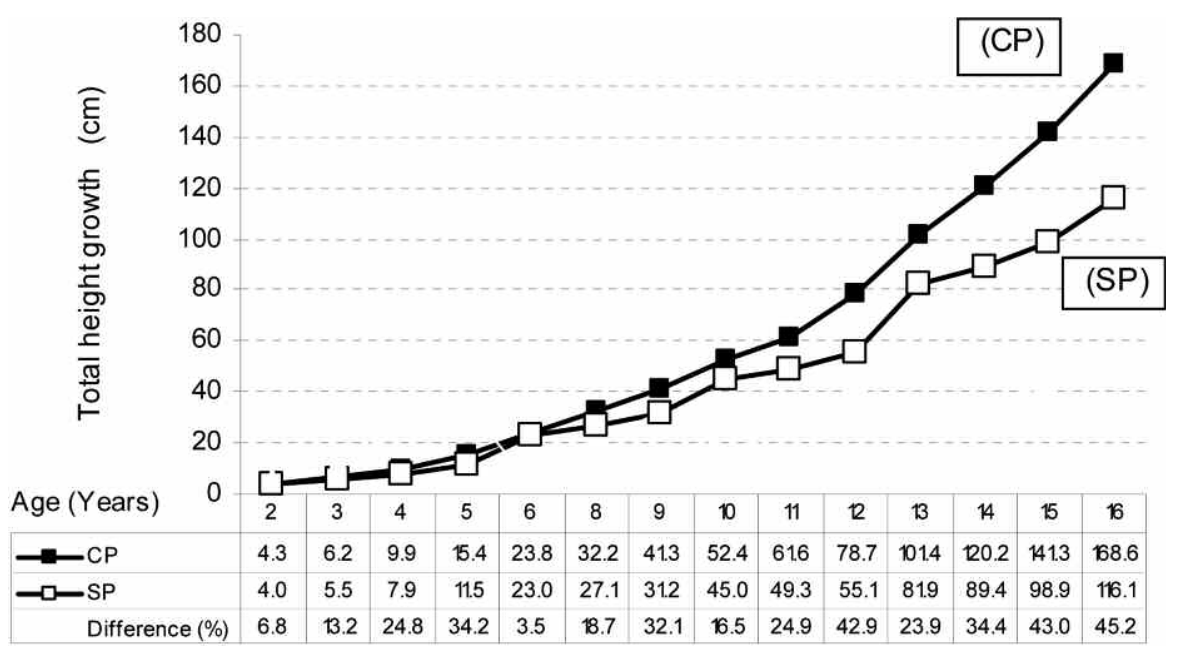

Figure 1. - Age trend in total height growth $(\mathrm{H})$ for mean control-cross-pollinated (CP) and mean control-self-pollinated (SP) progenies. 


\section{Variance components}

Across the field test, the $\sigma_{\text {GCA }}^{2}$ components of total height gradually increased with age from $45 \%$ to $65 \%$ while the estimates for annual height ranged, between $33 \%$ and $71 \%$, with no clear pattern (Table 3). According to SNYDER and NAMKOONG (1978), the variance component is deemed to be important, i.e. reliable, if it is estimated with a standard error less than half the magnitude of the variance. In this progeny test all $\sigma_{\text {GCA }}^{2}$ variance estimates for total and annual height have standard errors less than half the magnitude of the respective components, making them fairly reliable. In general, the $\sigma_{\text {SCA }}^{2}$ component estimates for total height ranged between nine and $14 \%$ and all their standard errors were less than half the magnitude of the respective component. Conversely, all $\sigma_{\text {SCA }}^{2}$ estimates for annual height are associated with standard errors higher than half the magnitude of the respective components, making them unreliable.

Most of both $\sigma^{2}{ }_{\text {Mat }}$ and $\sigma^{2}{ }_{\text {Mat-Int }}$ estimates for total and annual height have standard errors less than their variances: however, they do not fulfill the SNYDER's and NAMKOONG criterion. All but one variance component for survival have to be considered unreliable based on their large standard errors.

The time trend of the dominance ratios $\left(\sigma^{2} \mathrm{SCA} / \sigma^{2}{ }_{\mathrm{GCA}}\right)$ across both nursery and field tests for total and annual height traits are displayed in Figure 2. A continuous decreasing trend of the dominance ratios for total height was recorded; from 8.2 at age two to 0.3 at age 16 , while for annual height from 0.9 at age four to 0.2 at age 16 . Therefore, both additive and non-additive variances are present in this breeding population but the additive one is the more important source of variation for height.

\section{Heritability}

Broad-and narrow-sense full-sib family heritability estimates for total height increased with age and ranged within $0.65-0.76$ and $0.45-0.65$, respectively. Heritabilities reached the highest level between ages 14 and 16 years (Table 4). The same two heritabilities for annual height ranged within $0.47-0.76$ and $0.33-0.71$, respectively, but the time trend was irregular. Such trends could be attributable to variable climatic conditions across year, whose effect on annual growth is stronger

Table 3. - Estimates of genetic $\left(\sigma_{\mathrm{CGC}}^{2}, \sigma_{\mathrm{SCA}}^{2}, \sigma_{\text {Mat }}^{2}, \sigma_{\text {Mat-Inter. }}^{2}\right)$, environment $\left(\sigma_{\mathrm{e}}^{2}\right)$, within plot $\left(\sigma_{\mathrm{w}}^{2}\right)$ and plot error $\left(\sigma_{\mathrm{p}}^{2}\right)$ variances, standard errors and dominance ratios $\left(\sigma_{\mathrm{SCA}}^{2} / \sigma_{\mathrm{GCA}}^{2}\right)$. Genetic variances as percent in brackets were calculated relative to the phenotypic variance $\left(\sigma_{\text {ph.1 }}^{2}\right)$.

\begin{tabular}{|c|c|c|c|c|c|c|c|c|c|}
\hline \multirow[b]{2}{*}{ Components } & \multicolumn{9}{|c|}{ Trails / Estimales } \\
\hline & $\begin{array}{l}11.8 \\
(\mathrm{~cm})\end{array}$ & $\begin{array}{l}\mathrm{E} 1.9 \\
\text { (cm) }\end{array}$ & $\begin{array}{l}11.10 \\
(\mathrm{~cm})\end{array}$ & $\begin{array}{l}\text { H.I } \\
(\mathrm{cm})\end{array}$ & $\begin{array}{l}1.12 \\
(\mathrm{~cm})\end{array}$ & $\begin{array}{l}1.13 \\
(\mathrm{~cm})\end{array}$ & $\begin{array}{l}11.14 \\
(\mathrm{~cm})\end{array}$ & $\begin{array}{l}1.15 \\
(\mathrm{~cm})\end{array}$ & $\begin{array}{l}H .] 6 \\
(\mathrm{~cm})\end{array}$ \\
\hline \multirow{2}{*}{$\sigma^{2}{ }_{k C A}=S E$} & $4.127(45)$ & $6.894(52)$ & $11.704(52)$ & $14.030(55)$ & $27.448(60)$ & $41.274(63)$ & $59.247(65)$ & $77.091(64)$ & $95.189(65)$ \\
\hline & 12.036 & 13.245 & $=5.546$ & $: 6.624$ & 112.721 & $=18.903$ & 126.970 & $=35.531$ & 43.413 \\
\hline \multirow[t]{2}{*}{$\sigma^{2} \alpha_{A}: S L$} & $3.874(21)$ & $3.279(12)$ & $5.978(13)$ & $7.312(14)$ & $10.313(11)$ & $12.579(10)$ & $15.723(9)$ & $28.780(12)$ & $25.239(9)$ \\
\hline & \pm 1.187 & \pm 1.328 & $=2.417$ & $=2.786$ & $=4.447$ & \pm 5.718 & \pm 7.506 & $=11.607$ & $=12.425$ \\
\hline \multirow{2}{*}{ 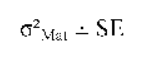 } & $0.613(3)$ & $0.349(1)$ & $0.793(2)$ & $1.162(2)$ & $2.929(3)$ & $4.469(3)$ & $6.050(3)$ & $4.969(2)$ & $7.332(3)$ \\
\hline & $\quad \perp 0.466$ & $\perp 0.454$ & -0.831 & -0.930 & -1.905 & $\perp 2.779$ & 13.687 & -3.568 & 14.984 \\
\hline \multirow[t]{2}{*}{$\sigma^{2} \mathrm{H}_{12-1}-111 \mathrm{SE}$} & $3.312(18)$ & $4.193(16)$ & $6.386(14)$ & $5.032(10)$ & $6.135(7)$ & $7.997(0)$ & $8.906(5)$ & $11.610(5)$ & $14.179(5)$ \\
\hline & \pm 1.043 & $\perp 1.518$ & -2.478 & -2.234 & -3.448 & $\perp 4.614$ & $\perp 5.880$ & -7.590 & $\perp 9.788$ \\
\hline \multirow[t]{2}{*}{$\sigma_{z}^{2} \perp \mathrm{SL}$} & $9.685(13)$ & $18.916(18)$ & $34.360(19)$ & $36.419(18)$ & $68.386(19)$ & $93.059(18)$ & $128.07(18)$ & $104.474(17)$ & $217.924(19)$ \\
\hline & $\perp 0.835$ & $\perp 1.631$ & -2.963 & -3.140 & -5.897 & 18.024 & $\perp 11.043$ & -14.182 & -18.791 \\
\hline$\sigma_{P}^{3} \mu_{L}:$ & 18.475 & 26.339 & 45.156 & 50.672 & 91.369 & 130.858 & 181.191 & 240.661 & 291.610 \\
\hline$\sigma_{H}$ & 26.680 & 48.812 & 84.344 & 117.504 & 185.980 & 340.152 & 401.693 & 562.306 & 625.484 \\
\hline \multirow{2}{*}{$\begin{array}{c}\sigma_{p}^{2} \\
\sigma_{p h ?}^{2}\end{array}$} & 7.017 & 14.035 & 25.932 & 24.669 & 49.788 & 59.044 & 87.900 & 108.243 & 155.376 \\
\hline & 49.751 & 84.456 & 146.840 & 183.740 & 310.040 & 506.789 & 638.766 & 870.091 & 1017.989 \\
\hline \multirow[t]{2}{*}{$\sigma_{l^{\prime} \|_{1, \hat{i}}}^{\prime}$} & 10.152 & 14.931 & 25.143 & 27.148 & 49.255 & 71.356 & 98.542 & 125.944 & 157.322 \\
\hline & \multicolumn{9}{|c|}{ Tahlo 3 (Continued) } \\
\hline \multirow[b]{2}{*}{ Comprouents } & \multicolumn{9}{|c|}{ lraits / Istimates } \\
\hline & $\begin{array}{c}b .9 \\
\text { (cons) }\end{array}$ & $\begin{array}{l}\text { b. } 10 \\
\text { (cmi) }\end{array}$ & $\begin{array}{l}\text { h.ll } \\
\text { (cmil) }\end{array}$ & $\begin{array}{l}\text { h.l2 } \\
\text { (ctn) }\end{array}$ & $\begin{array}{l}\text { h. } 13 \\
\text { (cm) }\end{array}$ & $\begin{array}{l}\text { h. } 14 \\
(\mathrm{~cm})\end{array}$ & $\begin{array}{l}h .15 \\
(\mathrm{~cm})\end{array}$ & $\begin{array}{l}\text { b. } 16 \\
(\mathrm{~cm})\end{array}$ & $\begin{array}{l}\text { SV.16 } \\
(\% .)^{\prime}\end{array}$ \\
\hline \multirow[t]{2}{*}{$\sigma^{*}{ }_{t i C A}-S E$} & $0.307(33)$ & $0.868(57)$ & $0.586 .(53)$ & $2.151(71)$ & $2.688(63)$ & $1.550(56)$ & $2.184(67)$ & $2.395(66)$ & $0.000(0)$ \\
\hline & $\therefore 0.170$ & $\perp 0.408$ & $\perp 0.278$ & $\perp 0.967$ & $\therefore 1.246$ & \pm 0.736 & \pm 0.998 & $=1.094$ & \pm 0.000 \\
\hline \multirow[t]{2}{*}{$\sigma^{2} 4 \mathrm{C}_{\mathrm{A}}=\mathrm{SE}$} & $0.268(14)$ & $0.225(7)$ & $0.067(3)$ & $0.232(4)$ & $1.068(13)$ & $0.630(11)$ & $0.575(9)$ & $0.513(7)$ & $5.093(30)$ \\
\hline & -0.170 & $+0,169$ & +0.126 & $+0,225$ & -0.436 & 10.328 & 10.295 & $: 0.324$ & 12.401 \\
\hline$\sigma^{2} \mathrm{yl}_{\mathrm{i}: 1}: \mathrm{SL}$ & $0.000(0)$ & $0.000(0)$ & $0.065(3)$ & $0.099(2)$ & $0.186(2)$ & $0.159(3)$ & $0.106(2)$ & $0.250(3)$ & $0.069(0)$ \\
\hline \multirow{3}{*}{$\sigma^{2} \mathrm{H}_{111} \mathrm{l}_{111}+\mathrm{SE}$} & $: 0.027$ & 10.032 & 10.049 & 10.060 & $=0.130$ & \pm 0.110 & \pm 0.083 & $=0.140$ & $\pm 0.34 !$ \\
\hline & $0.107(6)$ & $0,133(4)$ & $0.027(1)$ & $0.000(0)$ & $0.342(4)$ & $0.188(3)$ & $0.164(3)$ & $0.000(0)$ & $1.439(9)$ \\
\hline & $: 0.132$ & 10.147 & 10.116 & 10.150 & $: 0.267$ & 10.225 & 10.200 & 0.187 & 1.554 \\
\hline \multirow[t]{3}{*}{$\sigma_{c}^{*} \mid S E$} & $3.556(47)$ & $3.852(37)$ & $3.601(40)$ & $5.600(23)$ & $6.268(18)$ & $6.021(27)$ & $5.356(20)$ & $6.746(23)$ & $40.492(61)$ \\
\hline & -0.307 & $\perp 0.332$ & $\perp 0.310$ & $\perp 0.483$ & -0.510 & $\perp 0.519$ & 10.462 & $=0.582$ & $\perp 3.491$ \\
\hline & 1.878 & 3.063 & 2.231 & 6.033 & 8.539 & 5.581 & 6.551 & 7.239 & 16.724 \\
\hline$\sigma^{2}$ & 10.108 & 11.335 & 13.703 & 18.539 & 23.078 & 15.129 & 14,030 & 15,202 & 0.000 \\
\hline$\sigma^{2}$ & 2.545 & 2.719 & 2.230 & 3.746 & 3.960 & 4.508 & 3.953 & 5.225 & 40.492 \\
\hline 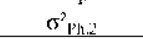 & 13.642 & 16.154 & 17264 & 26.918 & 34010 & 23.713 & 23.196 & 25.980 & 47.093 \\
\hline$\sigma_{P^{\prime} h, 3}^{2}$ & 0.923 & 1.610 & 1.200 & 3.215 & 4.417 & 2.891 & 3.429 & 3.811 & 7.135 \\
\hline
\end{tabular}

$\sigma_{\mathrm{Ph} 1}^{2}=2 \sigma_{\mathrm{GCA}}^{2}+\sigma_{\mathrm{SCA}}^{2}+\sigma_{\mathrm{Mat}}^{2}+\sigma_{\mathrm{Mat}-\mathrm{Int}}^{2}+\sigma_{\mathrm{e}}^{2} / \mathrm{k} ; \sigma_{\mathrm{Ph} 2}^{2}=2 \sigma_{\mathrm{GCA}}^{2}+\sigma_{\mathrm{SCA}}^{2}+\sigma_{\mathrm{Mat}}^{2}+\sigma_{\text {Mat-Int }}^{2}+\sigma_{\mathrm{P}}^{2}+\sigma_{\mathrm{W}}^{2} ; \sigma_{\mathrm{Ph} .3}^{2}=\sigma_{\mathrm{GCA}}^{2}+\sigma_{\mathrm{GCA}}^{2} /(\mathrm{p}-1)+$ $\left.\sigma_{\mathrm{SCA}}^{2} /(\mathrm{p}-1)+\sigma_{\text {Mat }}^{2}+\sigma_{\text {Mat-Int }}^{2}+\sigma_{\mathrm{e}}^{2} / 2 \mathrm{k}\right)$. H.8 to H.16 and h.9 to h.16 are the total and annual height between ages eight and 16 , respectively. 


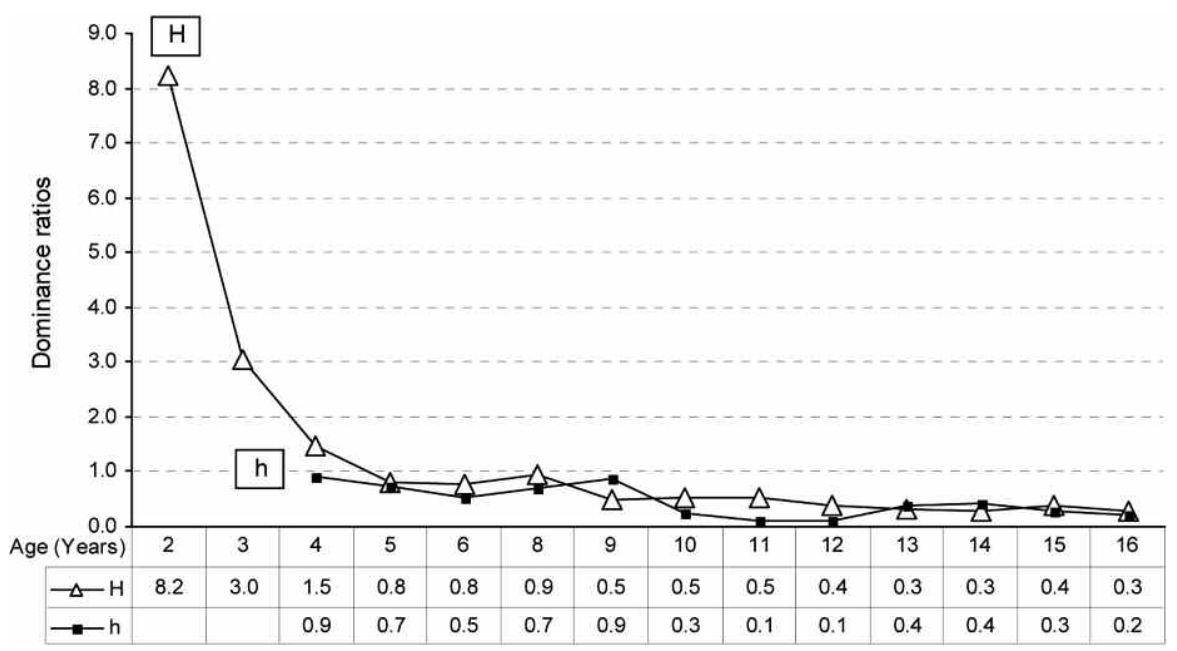

Figure 2. - Age trent of dominance ratios $\left(\sigma_{\mathrm{SCA}}^{2} / \sigma_{\mathrm{GCA}}^{2}\right)$ for total $(\mathrm{H})$ and annual $(\mathrm{h})$ height growth.

Table 4. - Estimates of phenotypic variances $\left(\sigma_{\mathrm{Ph} 1}^{2}, \sigma_{\mathrm{Ph} 2}^{2}, \sigma_{\mathrm{Ph} 3}^{2}\right)$, phenotypic standard deviations $\left(\sigma_{\mathrm{Ph} 1}, \sigma_{\mathrm{Ph} 2}, \sigma_{\mathrm{Ph} 3}\right)$, heritabilities $\left(\mathrm{h}_{\mathrm{b}-\mathrm{sFS}}^{2}, \mathrm{~h}_{\mathrm{n}-\mathrm{sFS}}^{2}, \mathrm{~h}_{\mathrm{n}-\mathrm{sHS}}^{2}, \mathrm{~h}_{\mathrm{n} \text {-s Ind }}^{2}\right)$ and the heritability standard errors $\left[\mathrm{SE}\left(\mathrm{h}_{\mathrm{b}-\mathrm{sFS}}^{2}\right) ; \mathrm{SE}\left(\mathrm{h}_{\mathrm{n}-\mathrm{sFS}}^{2}\right) ; \mathrm{SE}\left(\mathrm{h}_{\mathrm{n}-\mathrm{sHS}}^{2}\right) ; \mathrm{SE}\left(\mathrm{h}_{\mathrm{n}-\mathrm{s} \text { Ind }}^{2}\right)\right]$.

\begin{tabular}{|c|c|c|c|c|c|c|c|c|c|}
\hline & \multicolumn{9}{|c|}{ Traits / Estimates } \\
\hline Components & $\begin{array}{l}\text { H.8 } \\
(\mathrm{cm})\end{array}$ & $\begin{array}{l}\text { H.9 } \\
\text { (cm) }\end{array}$ & $\begin{array}{l}\text { H.10 } \\
(\mathrm{cm})\end{array}$ & $\begin{array}{l}\text { H.11 } \\
(\mathrm{cm})\end{array}$ & $\begin{array}{l}\text { H.12 } \\
(\mathrm{cm})\end{array}$ & $\begin{array}{l}\text { H.13 } \\
(\mathrm{cm})\end{array}$ & $\begin{array}{l}\text { H.14 } \\
(\mathrm{cm})\end{array}$ & $\begin{array}{l}\text { H.15 } \\
(\mathrm{cm})\end{array}$ & $\begin{array}{l}\text { H.16 } \\
(\mathrm{cm})\end{array}$ \\
\hline Mean & 32.2 & 41.3 & 52.4 & 61.6 & 78.7 & 101.4 & 120.2 & 141.3 & 168.6 \\
\hline$\sigma_{\mathrm{Phl}}^{2}$ & 18.475 & 26.339 & 45.156 & 50.672 & 91.369 & 130.858 & 181.191 & 240.661 & 291.610 \\
\hline$\sigma_{\mathrm{Ph} 2}^{2}$ & 49.751 & 84.456 & 146.840 & 183.740 & 310.040 & 506.789 & 638.766 & 870.091 & 1017.989 \\
\hline$\sigma^{2} \mathrm{Ph} 3$ & 10.152 & 14.931 & 25.143 & 27.148 & 49.255 & 71.356 & 98.542 & 125.994 & 157.322 \\
\hline$\sigma_{\mathrm{Phl}}$ & 4.298 & 5.132 & 6.720 & 7.118 & 9.559 & 11.439 & 13.461 & 15.513 & 17.077 \\
\hline$\sigma_{\mathrm{Ph} 2}$ & 7.053 & 9.190 & 12.118 & 13.555 & 17.608 & 22.512 & 25.274 & 29.497 & 31.906 \\
\hline$\sigma_{\mathrm{Ph} 3}$ & 3.186 & 3.864 & 5.014 & 5.210 & 7.018 & 8.447 & 9.927 & 11.225 & 12.543 \\
\hline $\mathrm{h}_{\mathrm{b}-\mathrm{SFS}}^{2}$ & 0.66 & 0.65 & 0.65 & 0.70 & 0.71 & 0.73 & 0.74 & 0.76 & 0.74 \\
\hline $\mathrm{SE}\left(\mathrm{h}_{\mathrm{b} \text {-s FS }}^{2}\right)$ & 0.28 & 0.30 & 0.30 & 0.32 & 0.33 & 0.33 & 0.34 & 0.34 & 0.34 \\
\hline $\mathrm{h}_{\mathrm{n}-\mathrm{S} F \mathrm{FS}}^{2}$ & 0.45 & 0.52 & 0.52 & 0.55 & 0.60 & 0.63 & 0.65 & 0.64 & 0.65 \\
\hline $\mathrm{SE}\left(\mathrm{h}_{\mathrm{n}-\mathrm{SFS}}^{2}\right)$ & 0.22 & 0.25 & 0.25 & 0.26 & 0.28 & 0.29 & 0.30 & 0.29 & 0.30 \\
\hline $\mathrm{h}_{\mathrm{n}-\mathrm{S} \mathrm{HS}}^{2}$ & 0.41 & 0.46 & 0.46 & 0.52 & 0.55 & 0.58 & 0.60 & 0.61 & 0.60 \\
\hline $\mathrm{SE}\left(\mathrm{h}_{\mathrm{n}-\mathrm{s} \mathrm{HS}}^{2}\right)$ & 0.20 & 0.22 & 0.22 & 0.24 & 0.26 & 0.26 & 0.27 & 0.28 & 0.28 \\
\hline $\mathrm{h}_{\mathrm{n}-\mathrm{s} \text { Ind }}^{2}$ & 0.33 & 0.33 & 0.32 & 0.30 & 0.35 & 0.33 & 0.37 & 0.35 & 0.37 \\
\hline $\mathrm{SE}\left(\mathrm{h}_{\mathrm{n}-\mathrm{s} \text { Ind }}^{2}\right)$ & 0.16 & 0.15 & 0.15 & 0.14 & 0.16 & 0.14 & 0.17 & 0.16 & 0.17 \\
\hline \multicolumn{10}{|c|}{ Table 4. (Continued) } \\
\hline & \multicolumn{9}{|c|}{ Traits / Estimates } \\
\hline Components & $\begin{array}{l}\text { h.9 } \\
(\mathrm{cm})\end{array}$ & $\begin{array}{l}\text { h.10 } \\
(\mathrm{cm})\end{array}$ & $\begin{array}{l}\text { h.11 } \\
(\mathrm{cm})\end{array}$ & $\begin{array}{l}\text { h.12 } \\
(\mathrm{cm})\end{array}$ & $\begin{array}{l}\text { h.13 } \\
(\mathrm{cm})\end{array}$ & $\begin{array}{l}\text { h.14 } \\
\text { (cm) }\end{array}$ & $\begin{array}{l}\text { h.15 } \\
(\mathrm{cm})\end{array}$ & $\begin{array}{l}\text { h.16 } \\
(\mathrm{cm})\end{array}$ & $\begin{array}{c}\text { SV.16 } \\
(\% .)\end{array}$ \\
\hline Mean & 9.4 & 13.1 & 12.7 & 19.8 & 24.8 & 19.4 & 23.7 & 28.2 & 98.7 \\
\hline$\sigma_{\mathrm{Phl}}^{2}$ & 1.878 & 3.063 & 2.231 & 6.033 & 8.539 & 5.581 & 6.551 & 7.239 & 16.724 \\
\hline$\sigma^{2} \mathrm{Ph} 2$ & 13.642 & 16.154 & 17.264 & 26.918 & 34.010 & 23.713 & 23.196 & 25.980 & 47.093 \\
\hline$\sigma_{\mathrm{Ph} 3}^{2}$ & 0.923 & 1.610 & 1.200 & 3.215 & 4.417 & 2.891 & 3.429 & 3.811 & 7.135 \\
\hline$\sigma_{\mathrm{Ph} 1}$ & 1.371 & 1.750 & 1.494 & 2.456 & 2.922 & 2.362 & 2.560 & 2.691 & 4.089 \\
\hline$\sigma_{\mathrm{Ph} 2}$ & 3.694 & 4.019 & 4.155 & 5.188 & 5.832 & 4.870 & 4.816 & 5.097 & 6.862 \\
\hline$\sigma_{\mathrm{Ph} 3}$ & 0.960 & 1.269 & 1.096 & 1.793 & 2.102 & 1.700 & 1.852 & 1.952 & 2.671 \\
\hline $\mathrm{h}_{\mathrm{b}-\mathrm{s} F \mathrm{SS}}^{2}$ & 0.47 & 0.64 & 0.55 & 0.75 & 0.75 & 0.67 & 0.75 & 0.73 & 0.13 \\
\hline $\mathrm{SE}\left(\mathrm{h}_{\mathrm{b}-\mathrm{s} \mathrm{FS}}^{2}\right)$ & 0.29 & 0.34 & 0.30 & 0.35 & 0.35 & 0.32 & 0.35 & 0.33 & 0.14 \\
\hline $\mathrm{h}_{\mathrm{n}-\mathrm{s} F \mathrm{~S}}$ & 0.33 & 0.57 & 0.52 & 0.71 & 0.63 & 0.55 & 0.67 & 0.66 & 0.00 \\
\hline $\operatorname{SE}\left(h_{n-S F S}^{2}\right)$ & 0.18 & 0.27 & 0.25 & 0.32 & 0.29 & 0.26 & 0.30 & 0.30 & 0.00 \\
\hline $\mathrm{h}_{\mathrm{n}-\mathrm{s} \mathrm{HS}}^{2}$ & 0.33 & 0.54 & 0.49 & 0.67 & 0.61 & 0.54 & 0.64 & 0.63 & 0.00 \\
\hline $\mathrm{SE}\left(\mathrm{h}_{\mathrm{n}-\mathrm{s} \mathrm{HS}}^{2}\right)$ & 0.18 & 0.25 & 0.23 & 0.30 & 0.28 & 0.25 & 0.29 & 0.29 & 0.00 \\
\hline $\mathrm{h}_{\mathrm{n}-\mathrm{s} \text { Ind }}^{2}$ & 0.09 & 0.21 & 0.14 & 0.32 & 0.32 & 0.26 & 0.38 & 0.37 & 0.00 \\
\hline $\mathrm{SE}\left(\mathrm{h}_{\mathrm{n}-\mathrm{s} \text { Ind }}^{2}\right)$ & 0.05 & 0.10 & 0.06 & 0.14 & 0.15 & 0.12 & 0.17 & 0.17 & 0.00 \\
\hline
\end{tabular}

H.8 to H.16 and h.9 to h.16 are the total and annual height growth between ages eight and 16, respectively.

than for total growth. Generally, the narrow-sense halfsib family means heritability estimates for total and annual growth traits are lower compared to the full-sib family heritabilities; they ranged from 0.41 to 0.61 and from 0.33 to 0.67 , respectively. Individual tree narrow- sense heritability estimates for total and annual height varied between 0.31 and 0.37 and between 0.09 and 0.38 , respectively.

All heritability estimates for total growth and all but six for annual growth are associated with standard 
errors less than half the magnitude of the respective estimates, i. e. they are reliable.

As the survival GCA variance was zero, only broadsense full-sib family heritability could be estimated; as expected, its value is very small (0.14) with a standard error greater than its respective estimate, i.e. not reliable.
These results indicate that heritability estimates are high enough to ensure genetic progress for improving growth of this Swiss stone pine population.

\section{General combining ability}

General combining ability (g.c.a.) effects calculated for each parent tree are listed in Table 5. Both positive and

Table 5. - Estimates of parent general combining ability effects (GCA).

\begin{tabular}{|c|c|c|c|c|c|c|c|c|c|}
\hline Progeryy & $\begin{array}{l}\mathrm{H} .8 \\
(\mathrm{~cm})\end{array}$ & $\begin{array}{l}\mathrm{H} .9 \\
(\mathrm{~cm})\end{array}$ & $\begin{array}{l}\mathrm{H} .10 \\
(\mathrm{~cm})\end{array}$ & $\begin{array}{l}\mathrm{H} .11 \\
(\mathrm{~cm})\end{array}$ & $\begin{array}{l}\mathrm{H} .12 \\
(\mathrm{~cm})\end{array}$ & $\begin{array}{l}\mathrm{H} .13 \\
\text { (cm) }\end{array}$ & $\begin{array}{l}H .14 \\
(\mathrm{~cm})\end{array}$ & $\begin{array}{l}\text { H.15 } \\
\text { (cm) }\end{array}$ & $\begin{array}{l}\mathrm{H} .16 \\
(\mathrm{~cm})\end{array}$ \\
\hline 2 & -0.947 & $-1.938 *$ & $-2.336^{*}$ & -1.645 & -1.997 & -1.356 & $-2,002$ & -2.414 & -1.640 \\
\hline 3 & $-1.564 * *$ & $-1.974 *$ & $-2.534 *$ & $-3.562 * * *$ & $-5.210 * * * *$ & $-5.700 * * *$ & $-6.508 * *$ & $-6.808 *$ & $-7.500 * *$ \\
\hline 45 & $-3.829 * * * *$ & $-4.605 * *$ & $-6.196 *$ & $-6.713^{* * * *}$ & $-9.108 * 4$ & 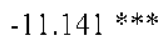 & $-13.175 * * *$ & $-14.880 * * *$ & $-15.617 \div * *$ \\
\hline 50 & 0.689 & 0.487 & 0.888 & 1.475 & 1.429 & 1.851 & 1.710 & 2,207 & 1.976 \\
\hline 205 & 0.389 & 1.088 & 0.514 & 0.056 & -1.200 & -2.246 & -3.859 & $-5.814 *$ & $-7.633 * *$ \\
\hline 206 & $1.457 * *$ & $2.026 * *$ & $3.340 * *$ & $2.870 * *$ & $3.275^{*}$ & $3.344^{*}$ & 3.843 & $5.364^{*}$ & 4.460 \\
\hline 209 & -1.050 & -0.903 & -0.770 & -0.880 & -0.511 & -1.818 & -0.413 & -0.817 & -1.352 \\
\hline $\mathrm{X}$ & $3.124 * * *$ & $3.434 * * * *$ & 4.107 *水* & $5.027 * * *$ & 7.960 *水* & $9.792 * * *$ & $12.612 * * *$ & $14.484 * * * *$ & $16.773^{* * *}$ \\
\hline $\bar{Y}$ & 0.251 & -0.256 & -0.501 & -0.279 & 1.000 & 1.264 & 1.974 & 3.135 & $5.187^{*}$ \\
\hline$\angle$ & $1.480 * *$ & $2.642 * * *$ & $3.489 * * *$ & $3.650 * * *$ & $4.363^{* *}$ & $6.010^{* * *}$ & $5.818 * *$ & $5.544 *$ & $5.346^{*}$ \\
\hline S.E. $\left(g_{;}-g_{j}\right)$ & 9.550 & 0.769 & 1.036 & 1.067 & 1.462 & 1.705 & 2.001 & 2.267 & 2.610 \\
\hline LSD $5 \%$ & 1.078 & 1.507 & 2.031 & 2.091 & 2865 & 3.342 & 3.921 & 4.444 & 5.115 \\
\hline$\angle S D / \%$ & 1.419 & 7.984 & 2.674 & 2.752 & 3.772 & 4.400 & 5.761 & 5.849 & 6.733 \\
\hline$\angle S D 0.1 \%$ & 1.810 & 2.529 & 3.409 & 3.510 & 4.810 & 5.610 & 6.582 & 7.459 & 8.586 \\
\hline \multicolumn{10}{|c|}{ Table 5 (Continued) } \\
\hline Progeny & $\begin{array}{c}\mathrm{h} .9 \\
(\mathrm{~cm})\end{array}$ & $\begin{array}{l}\mathrm{h}, 10 \\
(\mathrm{~cm})\end{array}$ & $\begin{array}{l}\mathrm{h}, 11 \\
(\mathrm{~cm})\end{array}$ & $\begin{array}{l}\mathrm{b}, 12 \\
(\mathrm{~cm})\end{array}$ & $\begin{array}{l}\text { h. } 13 \\
(\mathrm{~cm})\end{array}$ & $\begin{array}{l}\mathrm{h}, 14 \\
\text { (cm) }\end{array}$ & $\begin{array}{l}\text { h. } 15 \\
(\mathrm{~cm})\end{array}$ & $\begin{array}{l}\text { h. } 16 \\
(\mathrm{~cm})\end{array}$ & $\begin{array}{c}\text { SV.16 } \\
(\% .)\end{array}$ \\
\hline 2 & -0.554 & -0.387 & -0.053 & -0.078 & 0.199 & 0.238 & -0.255 & -0.159 & 0.821 \\
\hline 3 & -0.341 & $-0.706 *$ & -0.492 & -0.738 & -0.745 & -0.698 & -1.051 & -0.578 & 0.282 \\
\hline 45 & $-0.899 * *$ & $-1.573 * * *$ & $-1.356 * * *$ & $-2.382 * * *$ & $-3.059 * * *$ & $-2.129 * * * *$ & -2.040 *** & $-2.068 * * *$ & -0.217 \\
\hline 50 & 0.043 & 0.670 & 0.160 & -0.145 & 0.656 & 0.114 & 0.400 & 0.339 & 0.935 \\
\hline 205 & $0.731 *$ & $-0.694 *$ & -0.209 & $-1.195 * *$ & $-1.640 * * *$ & $-1.168 *$ & $-1.839^{* * * *}$ & $-2.201 * * *$ & -0.174 \\
\hline 206 & 0.625 & $1.112 * *$ & 0.060 & 0.137 & 0.301 & 0.277 & $1.124 * *$ & 0.005 & -0.244 \\
\hline 209 & -0.254 & -0.072 & -0.255 & 0.283 & 0.023 & 0.191 & 0.153 & 0.046 & -0.201 \\
\hline $\mathrm{X}$ & 0.331 & $0.742 *$ & $1,416^{* * * * *}$ & $2.590 * * * * *$ & $2,434 * * *$ & $2.259 * * *$ & $2.258 * * *$ & $2.379 * * *$ & 0.679 \\
\hline $\mathrm{Y}$ & -0.308 & -0.065 & 0.107 & 0.277 & $0.905^{*}$ & 0.739 & $1.169 * *$ & $1.605 * * *$ & 0.424 \\
\hline$Z$ & 0.625 & $0.974 * *$ & 0.621 & $1.250 * *$ & $0.926 *$ & 0.178 & 0.081 & 0.632 & $-2.304^{*}$ \\
\hline$S . E\left(g_{i}-g_{j}\right)$ & 0.333 & 0.347 & 0.335 & 0.478 & 0.443 & 0.434 & 0.409 & 0.459 & 1.725 \\
\hline$\angle S D 50$ & 0.653 & 0.680 & 0.657 & 0.820 & 0.867 & 0.850 & 0.802 & 0.900 & 2.205 \\
\hline$L S D 1 \%$ & 0.860 & 0.895 & 0.865 & 1.079 & 1.142 & 1.119 & 1.056 & 1.185 & 2.902 \\
\hline$\angle S D 0.1 \% / \mathrm{B}$ & 1.097 & 1.142 & 1.104 & 1.376 & 1.456 & 1.427 & 1.346 & $1.5 / 1$ & 3.701 \\
\hline
\end{tabular}

${ }^{*} \mathrm{p}<0.05 ;{ }^{* *} \mathrm{p}<0.01 ;{ }^{* * *} \mathrm{p}<0.001=$ significant and highly significant g.c.a. effects. H.8 to H.16 and h.9 to h.16 are the total and annual height growth between ages eight and 16 and nine and 16, respectively.

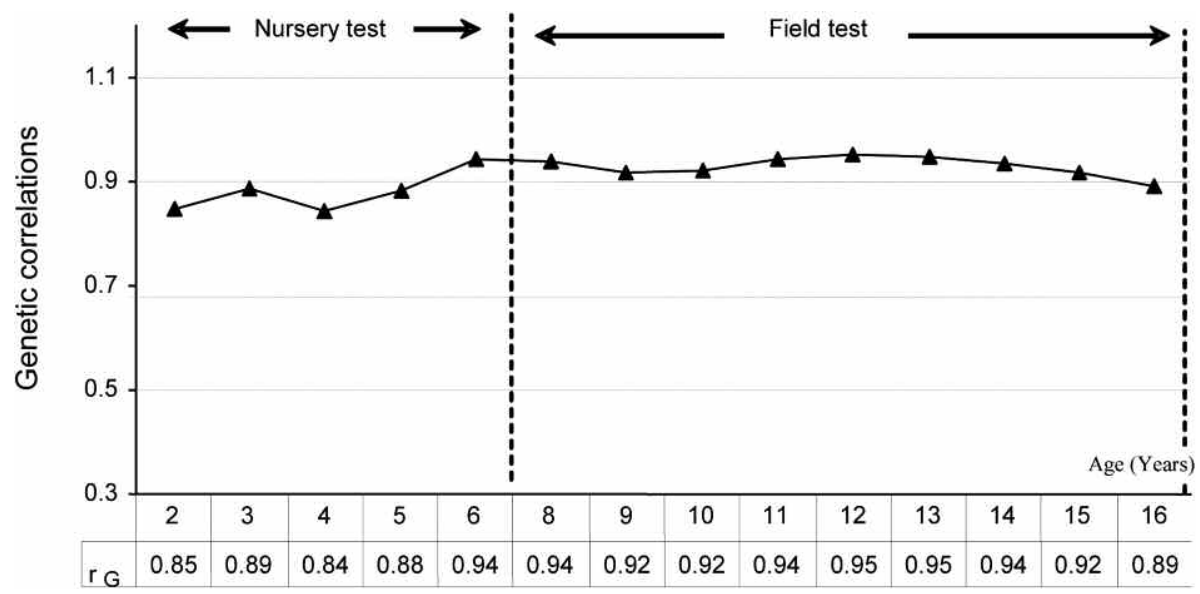

Figure 3. - Time trend of age-age genetic correlations $\left(\mathrm{r}_{\mathrm{G}}\right)$ across the whole testing period of total height growth. 


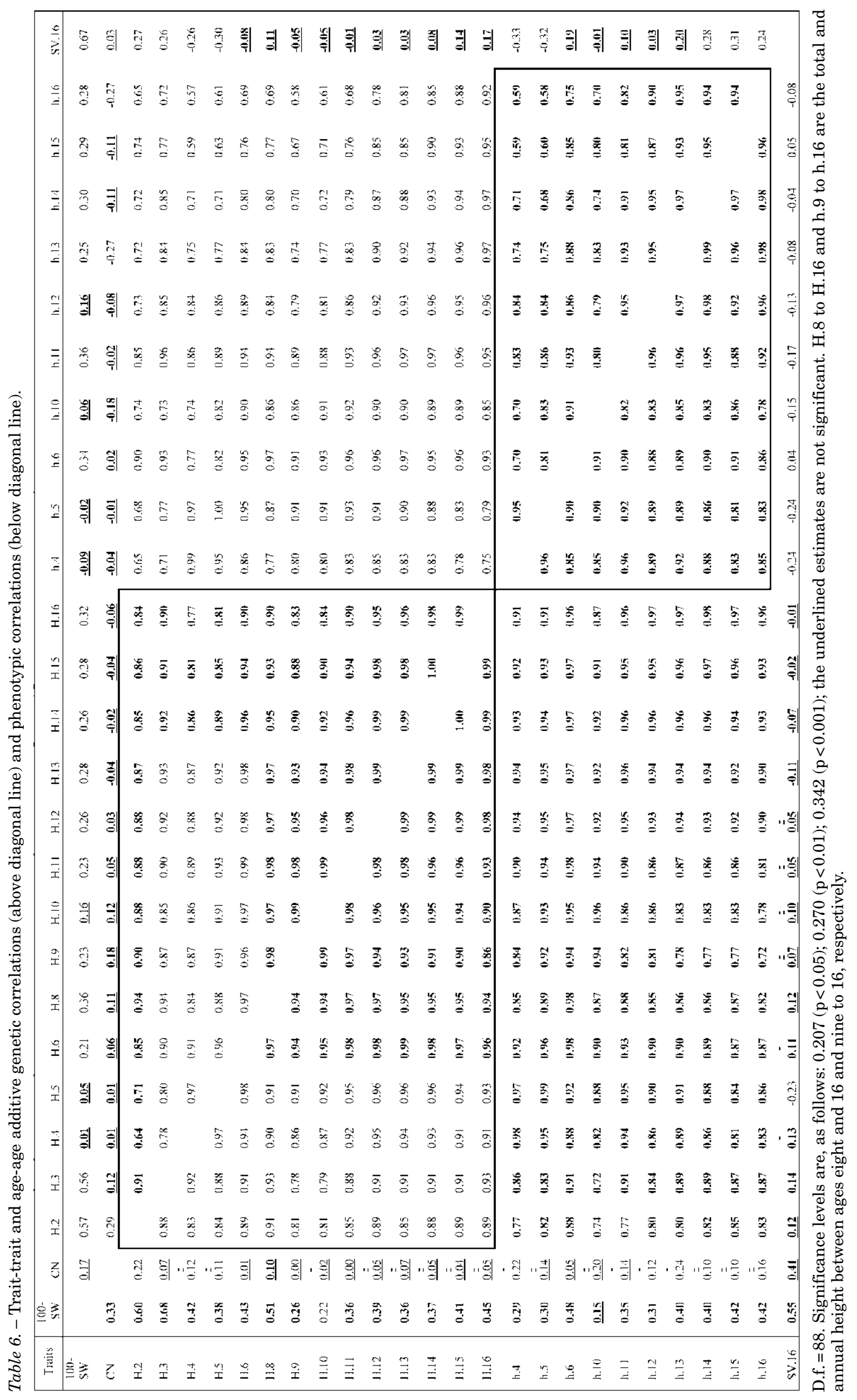


Table 7. - Expected genetic gain $(\Delta \mathrm{G} \%)$ that can be achieved in two growth traits.

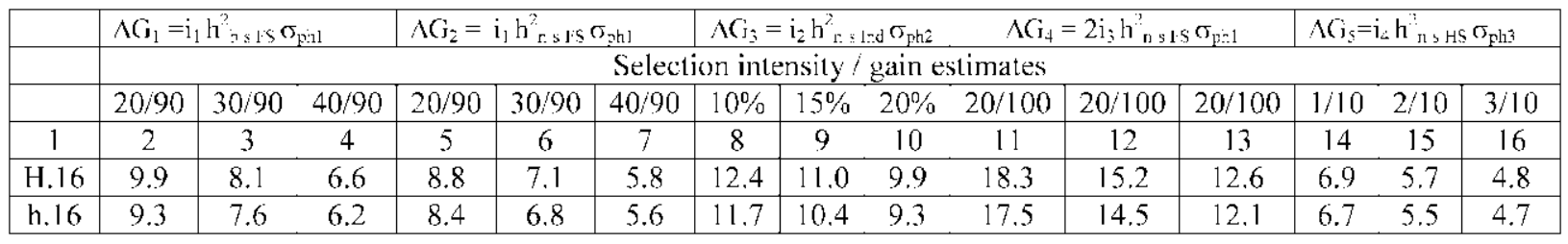

$\Delta \mathrm{G}_{1}$ and $\Delta \mathrm{G}_{2}=$ genetic gain if the best $20,30,40$ full-sib families are selected out of $90 ; \Delta \mathrm{G}_{3}=$ gain from mass selection if the best $10 \%, 15 \%, 20 \%$ individuals are selected within the best full-sib families; $\Delta \mathrm{G}_{4}=$ (scenario) gain from selection the best $20 ; 30$ or 40 GCA parents of 100 originated within the base population and intermated; $\Delta \mathrm{G}_{5}=$ genetic gain if the best 1 or 2 or, 3 half-sib families are selected out of 10 . H.16 and h.16 are the total and annual height to age 16 years.

negative significant and highly significant effects were found for both annual and total height. At all but one consecutive age, parent $X$ exhibited the largest significant or highly significant positive g.c.a. effects for total and annual height. Parent tree $Z$ is the second in rank; its g.c.a. effects were highly significant in all cases for total height and in three of eight cases for annual height, as well. Consequently, the $X$ and $Z$ parent trees should be promoted for further breeding work (hybridization) because of their high positive g.c.a. effects and high breeding value for total height. In contrast, parent trees 3 and 45 were the worst, showing highly significant negative g.c.a. effects for total and annual height growth. These two and all the other parents which have not shown positive significant effects should be disregarded for further breeding work including hybridization.

\section{Age-age genetic and phenotypic correlations} for the same trait

Very strong age-age additive genetic correlations were obtained between age 16 and previous ages for both total and annual height (Table 6). With minor exceptions (very early growth), all correlations for growth traits reached a high level approaching unity. The age-age additive genetic correlations ranged between 0.64 and 1.0 for total height and between 0.58 and 0.97 for annual height. Genetic correlations over time for total height increased from 0.85 at age two to 0.94 at age six and then leveled off across the field test until age 16 (Figure 3). Thus, this strong age-age genetic correlation for height indicates that if the goal is to improve 16-year height, early selection can be considered at age six.

Highly significant age-age phenotypic correlations at the full-sib family mean level were obtained across the whole testing period for both growth traits. They varied between 0.78 and 1.00 for total height and between 0.58 and 0.97 for annual height.

\section{Trait-trait genetic and phenotypic correlations}

The genetic correlation between 100 seed weight and cotyledon number was insignificant, while the phenotypic correlation was highly significant (Table 6). Genetic correlations between 100 seed weight and the total and annual height across the whole testing period were significant or highly significant for all but seven cases; but, phenotypic correlations at the family level between the same growth traits were highly significant for all but one case. Of particular interest, 100 seed weight was both genetically and phenotypically highly significantly correlated with survival at age 16 .

With one exception at age two, there were no significant genetic or phenotypic correlations between cotyledon number and the two height growth traits. The genetic correlation between cotyledon number and survival was not significant while the phenotypic one was highly significant. Highly significant trait-trait genetic and phenotypic correlations were obtained at successive ages between total and annual height; most of these estimates are closed to unity, but no consistent time trend was apparent.

\section{Selection and genetic gain}

The genetic gains calculated for the five selection strategies applied (Table 7) show that if the best 20, 30 or 40 out of the 90 tested full-sib families were selected and vegetatively propagated, a genetic gain $\left(\Delta \mathrm{G}_{1}\right)$ of $9.9 \%, 8.1 \%$ and $6.6 \%$, respectively, could be expected in total height; at the same intensity of selection but with the full-sib families propagated sexually, the gains $\left(\Delta \mathrm{G}_{2}\right)$ would be $8.8 \%, 7.1 \%$ and $5.8 \%$, respectively. With an intensity selection of $10 \%, 15 \%$ or $20 \%$ within the best full-families, a genetic gain $\left(\Delta \mathrm{G}_{3}\right)$ of $12.4 \%, 11.0 \%$ and $9.9 \%$, respectively, could be achieved.

If the best 20,30 or 40 of 100 GCA progeny are selected and intermated a genetic gain $\left(\Delta \mathrm{G}_{4}\right)$ of $18.3 \%, 15.2 \%$ and $12.6 \%$, respectively, could be made. Significant gains can be maximized if the improved material is used in large operational planting programs. With the selection of the best one or two or three of 10 half-sib families, the expected genetic gain $\left(\Delta \mathrm{G}_{5}\right)$ could be $6.9 \%, 5.7 \%$ and $4.8 \%$, respectively, i. e. this type of family selection achieves less gain than full-sib selection. Overall, the genetic gain that could be achieved in annual height was slightly smaller than that obtained for total height.

\section{Discussion}

\section{Variation in growth}

Swiss stone pine is a very slow growing species, especially at a young age. For example, at age six the annual and total mean height of the present progeny reached only 10.5 and $23.6 \mathrm{~cm}$, respectively (BLADA, 1999) and 28.2 and $168.6 \mathrm{~cm}$ respectively in the field test at age 16 (Table 4). Similarly, at six years of age, in a Swiss stone pine provenance test, total height showed very large 
variation, ranging from 10.2 to $27.5 \mathrm{~cm}$ with a mean of $16.8 \mathrm{~cm}$; the top provenance grew $64 \%$ faster than the trial mean and $170 \%$ faster than the slowest growing provenance (BLADA, 1997). Also, in an open pollinated half-sib progeny test of Swiss stone pine, highly significant differences among 136 families were found in total and annual height growth, root collar diameter and total number of branches (BLADA, 2003). Slow growth of Swiss stone pine was reported by HolzER (1975), as well. In comparison with Swiss stone pine, some other species grow much faster. For example, three year height was $101.1 \mathrm{~cm}$ in a Douglas-fir (Pseudotsuga menziesii (MIRB.) FrANCO) diallel progeny test (EL-KASSABY and PARK, 1993) and in a shortleaf pine (Pinus echinata MILL.) open-pollinated progeny/provenance test, mean stand height age 10 years varied across two locations between 6.0 and $7.5 \mathrm{~m}$ (TAUER and McNew, 1985).

At age 16, the outcrossed families of this progeny test out grew the selfed ones in total height by $45.2 \%$ (Figure 1), demonstrating that inbreeding depression occurred in this Swiss stone pine breeding population. Inbreeding depression was also reported in other conifer species, for example in Sitka spruce (Picea sitchensis Bong. CARR.) (SAMUEL, 1991) and black spruce (Picea mariana (MiLl.) B.S.P.) (MulLIN et al., 1992).

\section{Variances components}

It should noted that between age one and six of the nursery progeny test the dominance ratios for total height were in favor of the SCA variance (BLADA, 1999; Figure 2). In contrast, the time trend of the dominance ratio across the field test between age eight and 16 was in favor of GCA variance; at age 16 the GCA and SCA variances accounted for $65 \%$ and $9 \%$, respectively, of plot mean phenotypic variance (Table 3 ). At age six, in a half-sib family progeny test of the same Swiss stone pine species, the family additive genetic variance for total and annual height was $88 \%$ and $80 \%$, respectively (BLADA, 2003). An increase of additive variance with age for growth traits was also reported in radiata pine P. radiata D. Don (CoTTERILl et al., 1987; KING et al., 1998), in maritime pine (Pinus pinaster AIT.) (KUSNANDAR et al., 1998) and in loblolly pine (Pinus taeda L.) (Foster, 1986; McKeand and BRIDGwater, 1986; BALLOCHI et al., 1993; Li et al., 1996; GWAZE et al., 2001; XIANG et al., 2003a; XIANG et al., 2003b). In loblolly pine at age five both GCA and SCA were important sources of variation for growth traits (FosTER and BRIDGWATER, 1986). Greatly divergent patterns in the ratios of additive to dominance variance are evident as trees age. SAMUEL (1991) found that in Sitka spruce GCA variance component for height accounted for less than $2 \%$ of the total phenotypic variance while the SCA component accounted for between a quarter and a half at all assessments between age two to 15. PAWARAYI (1993) found that the amount of dominance in slash pine (Pinus elliottii ENGELM. var elliottii) becomes increasingly important at later ages. Conversely, this result is the exact opposite to what was found in a full-sib progeny test of the same slash pine species where dominance variance declined relative to additive variance with increasing age (DIETERs, 1995; DIETERS et al., 1995).

\section{Heritability}

At age six, in a half-sib family progeny test of Swiss stone pine, the family narrow-sense heritability for total and annual height grow and for root collar diameter was 0.97, 0.94 and 0.94, respectively (BLADA, 2003). In this study, a trend of a slow but constantly increasing heritability was recorded across nursery (BLADA, 1999) and the field test as well (Table 4), suggesting that this species offers good opportunities for genetic improvement of growth rate. This consistency in increasing heritability with time might be attributed not only to genetic factors, but also to environmental homogeneity in both nursery and field test, and perhaps because accurate measurements were made by the same technical team across the entire testing period.

There are no published estimates of heritability for growth traits for Swiss stone pine. However, similar trends in low or/and high heritability estimates for height growth were observed in other pine species. For example, BALOCCHI et al. (1993) and XIANG et al. (2003a) reported a similar increasing trend for tree height heritability estimates in loblolly pine and COSTA and DUREL (1996) in maritime pine. Also, a general increase with age of narrow-sense heritability in other conifers has been reported (MCKEAND, 1988; COTTERILL and DEAN, 1988; Hodge and White, 1992; BALOCCHI et al., 1993; JANSSON et al., 2003) and others have reported a non-linear, age-related trend (GWAZE and BRIDGEWATER, 2002) or that it is fairly constant with age (XIE and YING, 1996; SvENSSON et al., 1999; HAAPANEN, 2001). In contrast, for Pinus tecunumanii EG. \& PER. and P. chiapensis (MART.) ANDR. the heritability of height decreased with age (VASQUEZ and DvORAK, 1996) while in two studies with maritime pine, the time trend of the narrow sense heritabilities at family level for tree height were very constant over time (DANJON, 1994; KUSNADAR et al., 1998).

In this Swiss stone pine diallel study, a slowing down of the heritability increase was noticed the first 2-3 years after field planting; most likely due to the planting effects. Declining heritability during the early years after planting was also found in lodgepole pine (Pinus contorta Dougl.) (YING et al., 1989; XIE and YING, 1996) and seems to be a common phenomenon with transplanting conifers (JOHNSON et al., 1997).

\section{Correlations}

In this study, with minor exceptions, high and very high age-age additive genetic correlations over time for total height were obtained and the trend was increasing with age indicating that if the goal is to improve 16 -year height, early selection can be considered by age six. This relationship suggests that by age six the best g.c.a. parents and families can be selected and used in operational (planting) programs and in breeding for advanced generations. However later age correlations will be more reliable for final gain estimation. The age-age genetic correlations in this study were in close agreement with estimates for jack pine (Pinus banksiana Lamb.) (Riemenschneider, 1988, Magnussen and Yeatman, 1989). Early selections opportunities were reported in other species, as well. For example, the trends of heritabilities and age-age genetic correlations indicated that 
the optimum selection in loblolly pine could be as early as three for height and as early as age four for diameter at breast height and volume (Li et al., 1996; XIANG et al., 2003a). According to LAMBETH (1980) in Pinaceae and LAMBETH et al. (1983) in loblolly pine, adequate estimates of rotation age performance can be obtained at early ages, so most selection is currently done between the ages of five and ten years. Similarly, in an open pollination shortleaf pine progeny test, genetic correlations over time suggest that selection for age 10 volume using age two or four heights would be reliable (TAUER and MCNEW, 1985).

\section{General combining ability}

One of the aims of this study was to learn whether or not parent trees with high breeding value for use for seed orchard development can be selected within the present Swiss stone pine base population. As previously stated, two out of 10 randomly selected parent trees used in this diallel exhibited significant positive g.c.a. effects for height growth; consequently, by extrapolation it may be assumed that approximately $20 \%$ of trees within the base natural population might be good combiners. This high occurrence of good g.c.a. parent trees within the native base population suggests that selection of individuals for both seed orchard development and for advanced generation breeding populations is possible. Therefore, progeny testing of new candidates in order to detect enough good general combiners should be continued. While the initial parent selections in the base population were made at random, for new selections four traits will be taken into consideration: fast growth, stem straightness, crown density and disease free.

At present, most of the advanced tree improvement programs are based on good g.c.a. parent trees, for example the breeding programs in slash pine (HoDGE et al., 1991) and in loblolly pine (LI et al., 1996; Li et al., 1999; XIANG et al., 2003a). Based on these general observations, and the results from this study, a genetic improvement program with Swiss stone pine was launched in Romania with the following objectives: (i) phenotypic selection of parents in natural populations, (ii) testing provenances and open pollinated families, (iii) intra- and inter-specific controlled crosses, (iv) family testing and estimation of main genetic parameters followed by selection the best combiners and families, (v) seed-orchard establishment by using the best combiners in order to mass produce seed and to develop populations for advanced-generation breeding (BLADA, 1999). At present, crown density of Swiss stone pine is an important trait to improve because a dense crown has a high capacity for retention of both liquid and solid precipitation, thus reducing soil erosion, flooding and avalanche occurrence. Height growth and stem straightness are the next most important traits to be improved.

\section{Selection and genetic gain}

Generally, in a breeding program selection is based upon the principle that genetic value of selected families or individuals will be better than the average value of individuals in the population as a whole (ZOBEL and TALBERT, 1984). Table 7 shows that significant genetic gain in Swiss stone pine at age 16 , at both the family and the individual level can be achieved. By selecting full-sib families greater genetic gains were estimated than expected from half-sib family selection. The expected gain in height growth from selection based on intermated high g.c.a. parents could produce higher genetic gains than selection based on both full-sib families and on individuals within full-sib families; for example, selection of the $10 \%$ best individuals within full-sib families resulted in a gain of $12.4 \%$ compared to $18.3 \%$ when the best 20 of 100 g.c.a. parents are selected and intermated. It should be stressed that in this progeny test, parental selection was random with regard to growth and for this reason heritability and consequently genetic gains are underestimated. A similar case was reported by KRIEBEL (1983) in eastern white pine (Pinus strobus L.). XIANG et al. (2003b) reported that full-sib family selection in loblolly pine produced $40 \%$ genetic gain over selection on half-sib families while CARSON (1986) reported that in radiata pine, the highest gain could be made by selection and controlled intermating of high g.c.a. parents. The trends of age-age genetic correlations and heritabilities from this diallel progeny test indicate that selection can be considered at age six; this early selection is in agreement with LAMBETH (1980) and LAMBETH et al. (1983) for loblolly pine. The opportunity for early selection in some species, has been demonstrated, such as: in shortleaf pine (TAUER and McNEW, 1985), loblolly pine (LOWE and van BUIJTENEN, 1989; XIANG et al., 2003a), radiata pine (KUMAR and LEE, 2002), scotch pine (Pinus silvestris L.) (JANsson et al., 2003), lodgepole pine (XIE and YING, 1996); and maritime pine (KUSNADAR et al., 1998).

\section{Implications for Breeding Strategy}

The results of this study indicate that height of Swiss stone pine is under a strong genetic control and consequently, the species offers good opportunities for genetic improvement. Reliable data on the amount of GCA versus SCA genetic variance (Table 3; Figure 2) have already indicated reasonable genetic gains from a program that employs good g.c.a. selections. Taking into consideration that the GCA variance was the most important component of phenotypic variance, the breeding strategy of Swiss stone pine improvement will be based on recurrent selection for GCA variance. Applying this strategy is in line with similar strategies for slash pine (HoDGE et al., 1991; WHITE et al., 1993) and loblolly pine (LI et al., 1996; Li et al., 1999; XIANG et al., 2003a; JANSSON and LI, 2004).

To exploit the existing GCA variance from within the present Swiss stone pine population there will be applied a two stage method which involves selection of the best families followed by selection of individuals within families. This strategy works well with traits with lower heritability, such as growth rate, and it is the predominant form of selection used in most advanced generation tree improvement programs (ZOBEL and TALBERT, 1984). Therefore, a second generation clonal seed orchard of Swiss stone pine will be developed by using at least the best 200 individuals selected from within the best full-sib families. In addition, the breeding program 
is focusing on selection of new good general combiners to be used for development of control-pollinated seed orchards and advanced generation breeding population. Based on a study of radiata pine, CARSON (1986) concluded that production of improved seed through crossing among good general combiners is both an efficient and an effective strategy for optimizing gains from controlpollinated seed orchards. JANSSON and LI (2004) reported that selection based on full-sib families within disconnected diallels in loblolly pine only gave a small increase in gain compared to the selection based on parents that had high g.c.a. values within the same diallels; therefore, greater genetic gains should result from large scale production of the best general combiners, rather than selection and multiplication of the best full-sib families.

As previously shown in Table 3 , the SCA variance in the Swiss stone pine population significantly decreased from age eight until age 16 when it accounted for only $9 \%$ of phenotypic variance. Consequently, the relatively small importance of SCA in this population gives little merit to its exploitation. However, if a future increase in SCA variance is noted, then the present decision needs to be reconsidered.

\section{Conclusions}

- As significant genetic variation for growth traits was found, selection within Swiss stone pine should be effective.

- Across the field testing period, GCA variance was found to be more important than SCA, suggesting that the breeding strategy should be based on GCA.

- Age-age genetic correlations of early height increased significantly until age six (nursery test) then leveled off across the field test until age 16; the trends of age-age genetic correlations and heritabilities indicate that if the goal is to improve 16-year height, selection can be considered at age six.

- The highly significant variation in general combining ability for growth found in two of 10 tested parents suggested that at least $20 \%$ of trees in the base population should be good g.c.a. parents; such parents should be used: (i) for seed orchard development; (ii) in operational planting programs and (iii) for developing advanced-generation breeding populations.

- The genetic parameter estimates obtained in this study should help to make selection decisions to improve the current breeding program.

- Developing a breeding program for this slow growing species is recommended because of its major ecological and forestry importance for the Carpathian Mountains; in addition, the species is highly appreciated for (i) its valuable wood for furniture and sculpture, (ii) as source of resistance to Cronartium ribicola and (iii) for landscaping.

\section{Acknowledgements}

The authors gratefully acknowledge Professor C. TAUER from the Oklahoma State University (USA) and Dr. I. CABUlEA from the Agricultural Research Station, Turda (Romania) for their critical review of an ear- lier version of the manuscript. In addition, Professor C. TAUER significantly contributed to the English text improvement. Also the authors thank technical staff, i.e. S. Tanasie, A. Dragila and C. Dinu from the Forest Research and Management Institute for their contribution to the trial establishment and its periodically assessment. Thanks are extended to the unknown reviewer for valuable comments, suggestions and corrections on the manuscript, owing to which the article has gained in quality.

\section{Literature}

Anderson, R. L. and T. A. BANCROFT (1952): Statistical theory in research. McGraw-Hill Book Co., New-York, $399 \mathrm{p}$.

Ballochi, C. E., F. E. BRidgwater, B. J. Zobel and S. JAHROMI (1993): Age trends in genetic parameters for tree height in nonselected populations of loblolly pine. For. Sci. 39: 231-251.

BECKER, W. A. (1984): Manual of procedures in quantitative genetics. Fourth Edition. Acad. Enterp. Pullman, Washington, $190 \mathrm{p}$.

BELDIE, A. (1941): Observaţii asupra vegetaţiei lemnoase din Munţii Bucegi. Analele ICEF, Seria 1, Vol. 6: 39-43.

BINGHAM, R. T. (1972): Taxonomy, crossability and relative blister rust resistance of 5 needle pines, p. 271-280. In: Bingham, R. T., R. J. Hoff and G. I. McDonald (Eds.), Biology of rust resistance in forest trees. USDA For. Serv., Misc. Publ. 1921.

BlaDA, I. (1994): Interspecific hybridization of Swiss Stone Pine (Pinus cembra L.). Silvae Genetica 43 (1): 14-20.

Blada, I. (1995): Diallel cross in Pinus cembra. I: Results of the three years old nursery test. In: IUFRO Congress, Tampere, Finland, 6 to 12 August, Poster abstracts p. 66 .

BlaDA, I. (1996): Breeding of Pinus cembra and its nursery and planting operations. Annual Rep. No. 26, For. Res. Inst., Bucharest, $5 \mathrm{p}$.

BlaDA, I. (1997): Stone pine (Pinus cembra L.) provenance experiment in Romania: I. Nursery stage at age 6 , Silvae Genetica 46 (4): 197-200.

BladA, I. (1999): Diallel crossing in Pinus cembra. III: Analysis of genetic variation the nursery stage. Silvae Genetica 48 (3-4): 179-187.

BlaDA, I. (2003): Genetic variation in stone pine half-sib progenies, pp. 85-95. In: McKinley, C. R. (Ed.). Proc. $27^{\text {th }}$ Southern Forest Tree Improv. Conf., Stillwater, Oklahoma USA, June 24-27, 221 p.

BLADA, I. (2007): Swiss stone pine provenance experiment in Romania: II. variation in growth and branching traits at age 14. Silvae Genetica 56 (3-4): 148-158.

Blada, I. and F. Popescu (2008): Diallel Crossing in Pinus cembra. IV. Age Trends in Genetic Parameters and Genetic Gain for Growth and Branching Traits, pp. 89-113. Annals of Forest Research, Vol. 51, 180 p.

BReche, Y. and L. Chavautier (1975): Une vieille vallée raconte ses souvenirs. Petite histoire de la Tarantaise. Collection „Trsors de la Savoie“. Ed. Xavier Mappus, $83 \mathrm{p}$.

Carson, M. J. (1986): Control-pollinated seed orchards of the best general combiners - a new strategy for radiate pine improvement, pp. 144-149. In: WilliaMS, T. S. and G. S. WratT (Eds.). Proc. Plant Breeding Symposium DSIR 1986. Agron. Soc. of New Zealand Special Publ. No. 5. 
Contini, L. and Y. LaVAREllo (1982): Le pin cembro. INRA, Paris, $197 \mathrm{p}$.

Costa, P. and C. E. Durel (1996): Time trend in genetic control over height and diameter in maritime pine. Can. J. For. Res. 26: 1209-1217.

Cotterill, P. P., C. A. DeAn and G. VAN WyK (1987): Additive and dominance genetic effects in Pinus pinaster, $P$. radiata and $P$. elliottii and some implications for breeding strategy. Silvae Genetica 36 (5-6): 221-232.

Cotterill, P. P. and C. A. Dean (1988): Changes in the genetic control over height and diameter in maritime pine. Can. J. For. Res. 26: 1209-1217.

CRitchfield, W. B. and E. L. LitTLE JR. (1966): Geographic distribution of the pines of the world. U.S.D.A. For. Serv. Misc. Publ. No. 991.

DANJON, F. (1994): Heritability and genetic correlations for estimated growth curve parameters in maritime pine. Theor. Appl. Genet. 89: 911-921.

DiETERS, M. J. (1995): Genetic parameters for slash pine in south-east Queensland, Australia: growth, stem straightness and crown defects. Forest Genetics 3 (1) 27-36.

Dieters, M. J., T. L. White and G. R. Hodge (1995): Genetic parameter estimates for volume from full-sib tests of slash pine. Can. J. For. Res. 25: 1397-1408.

EL-KASSABY, Y. A. and Y. S. PARK (1993): Genetic variation and correlation in growth, biomass and phenology of Douglas-fir diallel progeny at different spacing. Silvae Genetica 42 (6): 289-297.

FALCONER, D. S. (1981): Introduction to quantitative genetics. Fourth edition, Longman and Co., New York, 340 p.

FALCONER, D. S. (1989): Introduction to quantitative genetics. $3^{\text {rd }}$ edition, Longman Scientific and Technical, Logman Group, Hong Kong.

FEKETE, L. es T. BLATTNY (1913): Az erdeszeti jelentosegu, fak es cserjek elterjedese: A magyar allam teruleten. Selmecbanya.

FosteR, G. S. (1986): Trends in genetic parameters with stand development and their influence on early selection for volume growth in loblolly pine. Forest Science 32: 944-959.

Foster, G. S. and F. E. BRIDGwater (1986): Genetic analysis of fifth-year data from a seventeen parent partial diallel loblolly pine. Silvae Genetica 35 (2-3): 118-122.

Georgescu, C. C. şi C. D. Ionescu-BArLad (1932): Asupra staţiunilor de Pinus cembra din Carpaţii Romaniei. Rev. Pad. 8-9: 531-543.

GRIFFING, B. (1956): Concept of general and specific combining ability in relation to diallel crossing systems. Australian J. Science 9: 463-493.

Gwaze, D. P., F. E. Bridgwater, T. D. Byram and W. J. LOWE (2001): Genetic parameter estimates for growth and wood density in loblolly pine. Forest Genetics 8 (1): 47-55.

Gwaze, D. P. and F. E. Bridgewater (2002): Determining the optimum selection age for diameter and height in loblolly pine. Forest Genetics 9: 159-165.

HAAPANEN, M. (2001): Time trends in the genetic parameter estimates and selection efficiency for Scots pine in relation to field testing method. Forest Genetics 8: 129-144.

HALlaueR, A. R. and J. B. MiRANDA (1981): Quantitative genetics in maize breeding. Iowa State University Press/Ames, $468 \mathrm{p}$.

Hodge, G., T. White and G. Powell (1991): Thirtythird progress report. Cooperative Forest Genetics Research Program, Dpt. of Forestry, Univ. of Forida, Gainesville.
Hodge, G. R and T. L. White (1992): Genetic parameter estimates for growth traits at different ages in slash pine and some implications for breeding strategy. Silvae Genetica 41: 252-262.

Hoff, R. J., R. T. Bingham and G. I. McDonald (1980): Relative blister rust resistance of white pines. Eur. Jour. Forest. Path. 10 (5): 307-316.

HoltmeieR, F.-K. (1994): Introduction to the Upper Engadine and its forests, pp. 9-17. In: SchMidT, W. C. and F.-K. Holtmeier (Eds.). Proc. Int. Workshop on Subalpine Stone Pines and their Environment: the Status of our Knowledge. USDA For. Serv., Int. Res. Station, Tech. Rep. INT-GTR-309, $321 \mathrm{p}$.

HolzER, K. (1969): Erste Ergebnisse der Auswahl von Zirbeneinzelbaumen (Pinus cembra L.). Centralblatt fur das Gesamte Forstwesen 86: 149-160.

Holzer, K. (1975): Genetics of Pinus cembra L. Annales Forestales, 6/5, Zagreb, $158 \mathrm{p}$.

Jannson, G., B. Li and B. HANnRUP (2003): Time trend in genetic parameter for height and optimal age for parental selection in Scotch pine. Forest Sci. 45: 696-705.

JANSson, G., B. Li and HANNRUP (2003b): Time trends in genetic parameters for height and optimum age for parental selection in Scots pine. Forest Sci. 49: 696-705.

JANSSON, G. and B. LI (2004): Genetic gain of full-sib families from disconnected diallels in loblolly pine. Silvae Genetica 53 (2): 60-64.

Johnson, G. R., R. A. Sniezko and N. L. MANDEL (1997): Age trends in Douglas-fir genetic parameters and implications for optimum selection age. Silvae Genetica 46 349-358.

KInG, J. N., M. J. CARson and G. R. Johnson (1998): Analysis of disconnected diallel mating designs. II: results from a third generation progeny test of the New Zealand Radiata pine improvement programme. Silvae Genetica 47 (2-3): pp. 80-87.

KRIEBEL, H. B. (1983): Breeding eastern white pine: a world-wide perspective. Forest Ecology and Management, 6: 263-279.

KRIEBEL, H. B., G. NAmkoong and R. A. Usanis (1972): Analysis of genetic variation in 1-, 2-, and 3-year old eastern white pine in incomplete diallel cross experiments. Silvae Genetica 21 (1-2): 44-47.

KuMAR, S. and J. LEE (2002): Age-age correlations and early selection for end-of-rotation wood density in Radiata pine. Forest Genetics 9 (4): 323-330.

Kusnandar, D., N. W. Galwey, G. L. Hertzler and T. B. Butcher (1998): Age trends in variances and heritabilities for diameter and height in Maritime pine (Pinus pinaster Ait.) in western Australia. Silvae Genetica 47 (2-3): 136-141.

LAMBETH, C. C. (1980): Juvenile mature correlations in Pinaceae and implications for early selection. Forest Sci. 36 (4): 571-580.

Lambeth, C. C., J. P. van BuiJtenen, S. D. Duke and R. B. McCullough (1983): Early selection is effective in 20-year-old-tests of loblolly pine. Silvae Genetica 32: 210-215.

LI, B., S. E. MCKEAND and R. J. WEIR (1996): Genetic parameters estimates and selection efficiency for the loblolly pine breeding in the south-eastern US. In: DIETERS, M. J. (Ed.), Tree improvement for sustainable forestry. QFRI-IUFRO Conf. Coloundra, Queensland, Australia.

LI, B., S. E. McKeand and R. J. WeIR (1999): Tree improvement and sustainable impact of two cycles of loblolly pine breeding in the U.S. Forest Genetics 6 (4): 229-234. 